Received 2012 OCtober 22; ACCEPted 2012 November 26; Published 2013 January 4

Preprint typeset using $\mathrm{LAT}_{\mathrm{E} X}$ style emulateapj v. 5/2/11

\title{
WIDE-FIELD MULTIBAND PHOTOMETRY OF GLOBULAR CLUSTER SYSTEMS IN THE FORNAX GALAXY CLUSTER
}

\author{
Hak-Sub Kim ${ }^{1}$, Suk-Jin Yoon ${ }^{1}$, Sangmo Tony Sohn ${ }^{2}$, Sang Chul Kim ${ }^{3}$, Eunhyeuk Kimª ${ }^{4}$, \\ Chul Chung ${ }^{1}$, SAng-Yoon LeE ${ }^{1}$, ANd Young-WoOK LeE ${ }^{1}$ \\ ${ }^{1}$ Department of Astronomy \& Center for Galaxy Evolution Research, Yonsei University, Seoul 120-749, Republic of Korea; \\ sjyoon@galaxy.yonsei.ac.kr \\ ${ }^{2}$ Space Telescope Science Institute, 3700 San Martin Drive, Baltimore, MD 21218, USA \\ ${ }^{3}$ Korea Astronomy and Space Science Institute, Daejeon 305-348, Republic of Korea \\ ${ }^{4}$ Korea Aerospace Research Institute, Daejeon 305-806, Republic of Korea \\ Received 2012 October 22; accepted 2012 November 26; published 2013 January 4
}

\begin{abstract}
We present wide-field multiband photometry of globular cluster (GC) systems in NGC 1399, NGC 1404, and NGC 1387 located at the central region of the Fornax galaxy cluster. Observation was carried out through $U, B, V$, and $I$ bands, which marks one of the widest and deepest $U$-band studies on extragalactic GC systems. The present $U$-band photometry enables us to significantly reduce the contamination by a factor of two for faint sources $\left(V_{0} \sim 23.5\right)$. The main results based on some 2000 GC candidates around NGC 1399, NGC 1404, and NGC 1387 are as follows: (1) the GC system in each galaxy exhibits bimodal color distributions in all colors examined, but the shape of color histograms varies systematically depending on colors; (2) NGC 1399 shows that the mean colors of both blue and red GCs become bluer with increasing galactocentric radius; (3) NGC 1399 shows overabundance of GCs in the directions of NGC 1404 and NGC 1387, indicating their ongoing interactions; and (4) NGC 1399 also exhibits a 0'.5 offset between the center of the inner GC distribution and the galaxy's optical center, suggesting that NGC 1399 is not yet dynamically relaxed and may be undergoing merger events.

Subject headings: galaxies: individual (NGC 1387, NGC 1399, NGC 1404) — galaxies: star clusters: general
\end{abstract}

\section{INTRODUCTION}

The Fornax galaxy cluster is the second nearest cluster of galaxies after Virgo, with a distance modulus of $(m-M)=31.51$ (Blakeslee et al. 2009) corresponding to a distance of $20.0 \mathrm{Mpc}$. It consists of two major components of the main Fornax cluster centered on NGC 1399 and a subcluster dominated by NGC 1316 (Drinkwater et al. 2001). There have been many photometric and spectroscopic studies of globular cluster (GC) systems in the central region of the Fornax cluster, and a fair number of them concentrated on the GC system associated with NGC 1399.

Bridges et al. (1991) and Wagner et al. (1991) presented the first CCD photometry for NGC 1399 GCs. Both studies presented an unusually high GC specific frequency of $S_{N}>15$ (i.e., number of GCs normalized to the galaxy luminosity; see Harris \& van den Bergh 1981). Although subsequent studies (Ostrov et al. 1998; Dirsch et al. 2003) derived a lower value $\left(S_{N} \sim 5\right)$, it is still higher than those of normal elliptical galaxies. Forbes et al. (1997) suggested that NGC 1404 GCs may have been tidally stripped by NGC 1399, resulting in a high $S_{N}$ value of NGC 1399 and a lower $S_{N}$ value of NGC 1404. Kissler-Patig et al. (1999) also proposed that the over abundance of NGC 1399 GCs can be explained by tidal stripping of GCs from neighboring galaxies and by the accretion of GCs in the gravitational potential of the Fornax cluster. Grillmair et al. (1999) supported this idea by showing that GCs in NGC 1399 and NGC 1404 are statistically indistinguishable in terms of their color distributions and luminosity functions in their $B, I$ photometry. Bekki et al. (2003) demonstrated the tidal stripping and accretion of GCs between NGC 1399 and NGC 1404 through their numerical simulations. Bassino et al. (2006a) presented the asymmetries in the azimuthal distribution of NGC 1399 GCs and suggested that they may be explained by the tidal stripping of GCs from NGC 1387.

Since Ostrov et al. (1993) first suggested a possible bimodal $C-T 1$ color distribution in the GC system of NGC 1399, many studies have confirmed the bimodal or multimodal GC color distribution in this galaxy through various passbands (e.g., Ostrov et al. 1998; Grillmair et al. 1999). Several studies have investigated the properties of blue and red GCs separately. Forbes et al. (1998) and Ostrov et al. (1998) showed that red GCs appear more centrally concentrated than blue GCs. Forte et al. (2005) suggested that the distribution of blue GCs is similar to that inferred for dark matter. Dirsch et al. (2003) presented that the surface density profile of blue GCs is shallower than that of red GCs within $8^{\prime}$ but they are not distinguishable beyond the radius. They also showed that the specific frequency of blue GCs is a factor of three larger than that of the red GCs within $7^{\prime}$. Forte et al. (2005) suggested that the specific frequencies for red and blue GCs are 3.3 and 14.3, respectively. Grillmair et al. (1999) showed that the peak of luminosity function for blue GCs is $0.36 \mathrm{mag}$ brighter in $B$ than that for red GCs. Blakeslee et al. (2012) found that the opticalinfrared color distribution of NGC 1399 GCs is unimodal, whereas the optical colors of the GCs exhibit bimodality. Forte et al. (2007) investigated the connection between GCs and the stellar population of NGC 1399 using their 
TABLE 1

Host Galaxy Properties

\begin{tabular}{ccccc}
\hline \hline Galaxy & $\begin{array}{c}\text { R.A. (J2000) } \\
\text { (h:m:s) }\end{array}$ & $\begin{array}{c}\text { Decl. (J2000) } \\
\left({ }^{\circ}:^{\prime}:^{\prime \prime}\right)\end{array}$ & $\begin{array}{c}B_{T} \\
(\mathrm{mag})\end{array}$ & Type \\
\hline NGC 1387 & $03: 36: 56.84$ & $-35: 30: 23.85$ & 12.3 & SB0 \\
NGC 1389 & $03: 37: 11.67$ & $-35: 44: 39.73$ & 12.8 & SB0 \\
NGC 1399 & $03: 38: 29.14$ & $-35: 27: 02.30$ & 10.6 & E0 \\
NGC 1404 & $03: 38: 52.08$ & $-35: 35: 37.67$ & 10.9 & E2 \\
& & & & \\
\hline
\end{tabular}

$C-T 1$ photometry. They suggested that the metal-poor subpopulation is homogeneous and exhibits an extended spatial distribution contributing $\sim 20 \%$ of the total stellar mass, while the metal-rich subpopulation is heterogeneous and dominates the inner region of the galaxy.

Recent studies based on the Advanced Camera for Surveys (ACS) Fornax Cluster Survey (FCS; Jordán et al. 2007) have looked into various properties of GC systems in the Fornax galaxy cluster. Mieske et al. (2010) detected color-magnitude relation for GCs (i.e., blue tilt) and found that the slope is shallower than that found for Virgo Cluster Survey GC samples. Masters et al. (2010) measured the half-light radii of GCs in ACS FCS galaxies, and suggested the mean half-light radius as a distance indicator. Villegas et al. (2010) found that the dispersion of the GC luminosity functions varies systematically with the brightness of parent galaxies. Liu et al. (2011) investigated the radial color gradients of GC systems in early-type galaxies in the Fornax galaxy cluster, and found that both red and blue GC subpopulations exhibit significant color gradients. They also found that the slope of the GC color gradients depends on mass of host galaxies.

There have been a few studies for the GC systems of NGC 1404 and NGC 1387. For NGC 1404, Richtler et al. (1992) presented $V, R$ photometry of the GC system, and derived a low specific frequency of $S_{N}=2.3$. They suggested that NGC 1404 may have its origin outside the Fornax cluster based on the low specific frequency similar to that of isolated galaxies together with its high radial velocity. Forbes et al. (1998) presented broad $B-I$ distributions for GCs in the inner and outer regions of NGC 1404 and tentatively suggested a presence of two GC subpopulations. The bimodal distribution in $B-I$ was later confirmed by Grillmair et al. (1999) and Larsen et al. (2001). For NGC 1387, Bassino et al. (2006b) found that the $C-T 1$ distribution of the GCs presents a distinct separation between red and blue GCs, and the radial distribution of red GCs is more centrally concentrated than blue GCs.

Several studies have been done on kinematics and dynamics for GCs around NGC 1399. Grillmair et al. (1994) suggested that, based on the low-dispersion spectra of 47 GCs, the large velocity dispersion of the GCs indicates that they are associated with the gravitational potential of the Fornax cluster rather than with that of NGC 1399. Kissler-Patio et al. (1999) supported this suggestion by finding a strong radial increase of the velocity dispersion for 74 GCs. They found no compelling evidence for rotation and could not detect differences between the kinematics of the blue and red GCs. Richtler et al. (2004) obtained medium-resolution spectra of $468 \mathrm{GCs}$, and they did not find any signature of
TABLE 2

OBSERVATION LOG

\begin{tabular}{lcccc}
\hline \hline Date (UT) & Filter & $\begin{array}{c}\text { Exposure Time } \\
(\mathrm{s})\end{array}$ & $\begin{array}{c}\text { Number of } \\
\text { Frames }\end{array}$ & $\begin{array}{c}\text { Seeing } \\
(\operatorname{arcsec})\end{array}$ \\
\hline 2006 Nov 24 & $U^{\mathrm{a}}$ & 1800 & 6 & $0.81-1.08$ \\
2006 Nov 24 & $B$ & 900 & 3 & $0.91-1.20$ \\
2006 Nov 24 & $V$ & 240 & 3 & $0.94-1.10$ \\
2006 Nov 24 & $I$ & 300 & 6 & $0.63-0.80$ \\
2006 Nov 28 & $U^{\mathrm{a}}$ & 1800 & 9 & $1.14-1.43$ \\
2006 Nov 28 & $B$ & 900 & 3 & $1.15-1.35$ \\
2006 Nov 28 & $V$ & 360 & 3 & $0.86-1.07$
\end{tabular}

${ }^{a}$ We used the SDSS $u$ filter and later calibrated the photometry to the Johnson $U$ system.

rotation. They suggested that the large velocity dispersion of the GCs could arise from the contamination by foreground objects. Schuberth et al. (2010) investigated kinematical and dynamical properties of the GC system of NGC 1399 using the spectra of some 700 GCs. They found that blue and red populations are kinematically distinct, and the properties of red population resemble those of field stellar populations.

Several spectroscopic studies investigated chemical abundance and ages of NGC 1399 GCs. Kissler-Patig et al. (1998) obtained spectra of 18 GCs in NGC 1399 and found that their abundances span the range observed in the Milky Way and M31. They pointed out that $V-I$ correlates well with metallicity but the slope of the relation becomes flatter toward redder colors if the values of Milky Way GCs are considered together. Forbes et al. (2001) investigated the ages of the GCs and found that the majority of their sample (10 GCs) have ages of around 11 Gyr.

In this paper we provide a new $U, B, V$, and $I$ photometric catalog of $\mathrm{GC}$ candidates in the central region of the Fornax galaxy cluster. This is one of the widest and deepest $U$-band studies on extragalactic GC systems. Section 2 describes the observations and data reduction. Section 3 gives the GC catalog for NGC 1399, NGC 1404, and NGC 1387, discussing the selection method and the foreground and background contamination level. Section 4 presents the properties of GC systems around the galaxies, including spatial distributions, color-magnitude diagrams (CMDs), color distributions, and the radial variations of color bimodality. Section 5 summarizes our results.

\section{OBSERVATIONS AND DATA REDUCTION}

\subsection{Observations}

The observations were carried out on the nights of 2006 November 24 and 28 (UT) with the Mosaic II CCD imager mounted on the prime focus of the $4 \mathrm{~m}$ Blanco telescope at Cerro Tololo Inter-American Observatory (CTIO). The observing conditions were clear and photometric with an average seeing of around $1^{\prime \prime}$. The Mosaic II consists of eight $2048 \times 4096$ pixel CCDs with a pixel scale of $0^{\prime \prime} .27$ providing a total field of view of $36^{\prime} \times 36^{\prime}$.

Our target field was chosen such that the brightest elliptical galaxy in the Fornax cluster, NGC 1399, and its three neighboring galaxies, NGC 1404, NGC 1387, and NGC 1389, were all imaged in a single telescope pointing. Figure 1 shows the median combined image of our target field as imaged in the $B$-filter, and Table 1 lists 


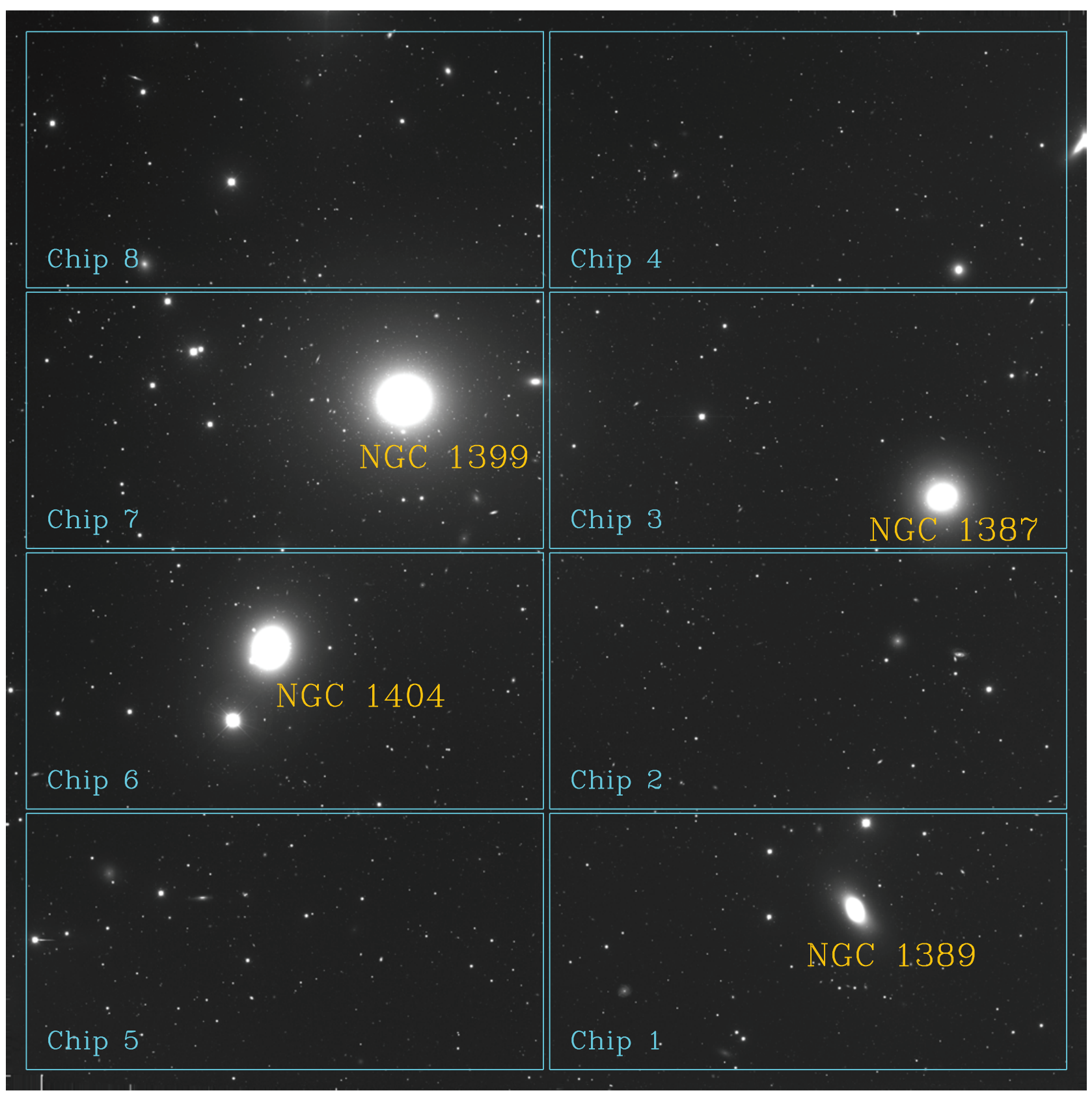

FIG. 1. - Median combined $B$-band image of our target field with the configuration of Mosaic II chips overlaid. The gaps between chips were filled in by creating mosaics using a three-point dither pattern. The image covers an area of $36^{\prime} \times 36^{\prime}$, containing four Fornax cluster galaxies as labeled and their GC systems. North is up, and east is to the left.

the basic properties of the four members of the Fornax cluster. We observed the target field with $u, B, V$, and $I$ filters. We note that the standard Johnson $U$ filter was not available at the time of our observations, so we used the Sloan Digital Sky Survey (SDSS) $u$ filter as an alternative and later calibrated the photometry to the Johnson $U$ system. The resulting effect of this calibration procedure is discussed below. The total exposure times in $u, B, V$, and $I$ were $27000,5400,1800$, and $1800 \mathrm{~s}$, respectively. We split our observations in each band into two sets of three dithered exposures (five sets for $u$ ) to fill in the gaps between the individual CCD chips in the mosaic, and to minimize the impact on our photometry from CCD blemishes (e.g., hot pixels and bad columns).
A summary of observations is provided in Table 2

\subsection{Pre-processing and Photometry}

All images were pre-processed using the MSCRED package (Valdes 1998) in IRAF W We used the CCDPROC task to correct for cross-talk between the CCD chips, apply and trim the overscan, correct for the bias level, and apply the flat-field correction. We used MSCPIXAREA to correct for the variations in pixel scales across the frame produced by geometric distortion

${ }^{1}$ IRAF is distributed by the National Optical Astronomy Observatory, which is operated by the Association of Universities for Research in Astronomy (AURA) under cooperative agreement with the National Science Foundation. 


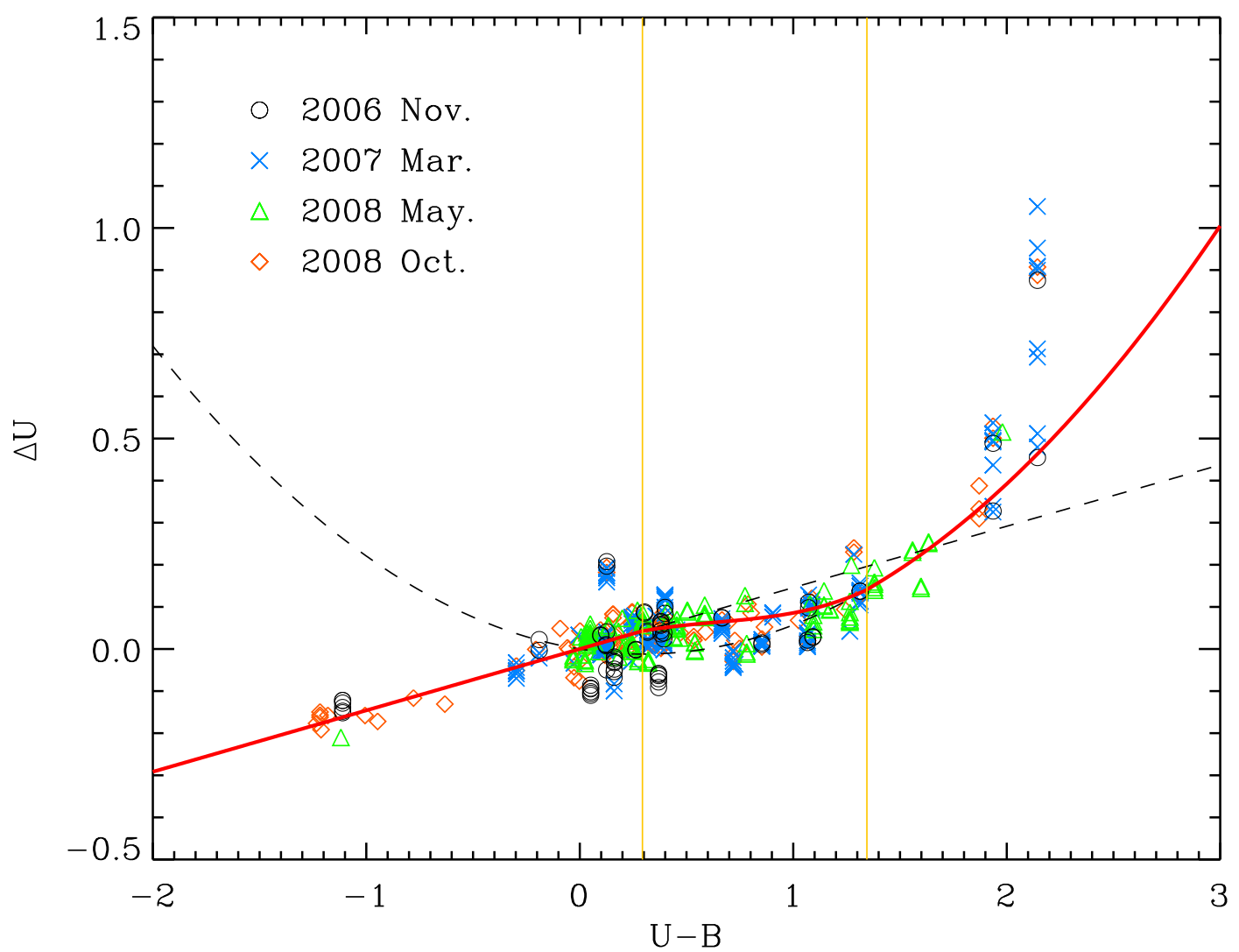

FIG. 2.- Difference between the standard and instrumental $U$ magnitudes of standard stars after photometric zero point and atmospheric extinction corrections, as a function of $U-B$. In the calibration of $U$ band, all the standard stars observed in subsequent observing runs during three years were used to derive a reliable solution. Different symbols are used to distinguish observations from different seasons. Two dashed lines represent error-weighted least-squares fits for blue and red standard stars, respectively. Red solid line represents the adopted best-fit function, which connects the two functions using internal division points of them between two vertical orange lines.

in the Mosaic II imager. Each mosaic image was then split into eight individual images using the MSCSPLIT task.

To produce a clean high signal-to-noise image in each filter, we combined the images using the MONTAGE2 routine in the ALLFRAME package (Stetson 1994). We then applied a ring median filter (Secker 1995) having an inner radius of 2.5 times the mean full width at half-maximum (FWHM) and a width of 5 pixel on the combined images and subtracted off these images from the original images to remove the rapidly varying background light of the galaxies while preserving the pointsources. Objects were detected from the backgroundsubtracted images using the DAOPHOT II/ALLSTAR routines through the following steps. First, we derived a list of objects and created new images with these objects subtracted. From these images, we detected fainter objects that were missed in the previous step and created another set of object-subtracted images. This procedure was iterated five times, and all detected objects were merged into a master catalog. The master catalog was then input to the ALLFRAME program (Stetson 1994) along with all of the images and their pointspread function (PSF) models for final PSF photometry. Aperture corrections were computed through the DAOGROW routine (Stetson 1990) using bright and iso- lated point-sources, and we applied them to our photometry above. Finally, an error-weighted mean instrumental magnitude for each object was obtained using the DAOMATCH/DAOMASTER routines (Stetson 1993).

The photometric calibration was achieved using standard stars in the fields of Landolt (1992) and Stetson (2000). During the course of our observing runs, we imaged eight standard fields placing the stars of interest on chip 6. Each image contains 15-25 standard stars, which covers an appropriately large range in color. We also observed the standard fields in many different airmasses. In the calibration of $U$ band, however, we used all the standard stars that are observed in subsequent observing runs during 2006, 2007, and 2008, because the photometric uncertainty in $U$ band is quite large and the number of standard stars is too small to derive reliable solutions. To calibrate the instrumental magnitudes to the Johnson-Cousins standard magnitudes, we used the transformation equation of the form

$M_{\text {std }}=m_{\text {inst }}+\mathrm{c} 1+\mathrm{c} 2 \times$ airmass $+\mathrm{c} 3 \times$ color $+\mathrm{c} 4 \times$ color $^{2}$,

where $M_{\text {std }}$ is the calibrated magnitude, $m_{\text {inst }}$ is the instrumental magnitude that is normalized to $1 \mathrm{~s}, \mathrm{c} 1$ is the photometric zero point offset, $\mathrm{c} 2$ is the extinction coefficient for airmass corrections, and both c3 and c4 are 
TABLE 3

Photometric Calibration Coefficients

\begin{tabular}{cccccc}
\hline \hline Date (UT) & Filter & c1 & c2 & c3 & c4 \\
\hline 2006 Nov 24 & $U_{\text {blue }^{a}}{ }^{a}$ & $-2.1194 \pm 0.0127$ & $-0.4495 \pm 0.0072$ & $0.1458 \pm 0.0038$ & NaN \\
& $U_{\text {red }}{ }^{a}$ & $-2.0699 \pm 0.0380$ & $-0.4442 \pm 0.0144$ & $-0.0819 \pm 0.0581$ & $0.1390 \pm 0.0259$ \\
& $B$ & $0.3711 \pm 0.0045$ & $-0.2236 \pm 0.0037$ & $0.0803 \pm 0.0015$ & NaN \\
& $V$ & $0.6402 \pm 0.0035$ & $-0.1386 \pm 0.0028$ & $-0.0297 \pm 0.0011$ & NaN \\
& $I$ & $0.0578 \pm 0.0039$ & $-0.0576 \pm 0.0030$ & $-0.0099 \pm 0.0011$ & NaN \\
2006 Nov 28 & $U_{\text {blue }}{ }^{a}$ & $-2.1641 \pm 0.0128$ & $-0.4495 \pm 0.0072$ & $0.1458 \pm 0.0038$ & NaN \\
& $U_{\text {red }}{ }^{a}$ & $-2.1196 \pm 0.0383$ & $-0.4442 \pm 0.0144$ & $-0.0819 \pm 0.0581$ & $0.1390 \pm 0.0259$ \\
& $B$ & $0.3243 \pm 0.0048$ & $-0.2236 \pm 0.0037$ & $0.0803 \pm 0.0015$ & NaN \\
& $V$ & $0.6061 \pm 0.0037$ & $-0.1386 \pm 0.0028$ & $-0.0297 \pm 0.0011$ & NaN \\
& $I$ & $0.0350 \pm 0.0041$ & $-0.0576 \pm 0.0030$ & $-0.0099 \pm 0.0011$ & NaN
\end{tabular}

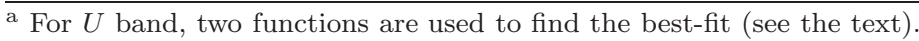

TABLE 4

Chip-To-Chip CORRECTION CoEFFICIENTS

\begin{tabular}{|c|c|c|c|}
\hline Chip & Filter & c1 & $\mathrm{c} 2$ \\
\hline Chip 1 & $\begin{array}{l}U \\
B \\
V \\
I\end{array}$ & $\begin{array}{c}0.0347 \pm 0.0020 \\
0.0236 \pm 0.0007 \\
-0.0165 \pm 0.0004 \\
0.0037 \pm 0.0005\end{array}$ & $\begin{array}{c}-0.0334 \pm 0.0055 \\
-0.0147 \pm 0.0022 \\
0.0029 \pm 0.0014 \\
0.0010 \pm 0.0017\end{array}$ \\
\hline Chip 2 & $\begin{array}{l}U \\
B \\
V \\
I\end{array}$ & $\begin{array}{l}0.0323 \pm 0.0019 \\
0.0162 \pm 0.0007 \\
0.0034 \pm 0.0005 \\
0.0023 \pm 0.0005\end{array}$ & $\begin{array}{c}-0.0609 \pm 0.0055 \\
-0.0094 \pm 0.0024 \\
0.0046 \pm 0.0015 \\
-0.0037 \pm 0.0017\end{array}$ \\
\hline Chip 3 & $\begin{array}{l}U \\
B \\
V \\
I\end{array}$ & $\begin{array}{l}0.0087 \pm 0.0019 \\
0.0197 \pm 0.0007 \\
0.0020 \pm 0.0004 \\
0.0085 \pm 0.0005\end{array}$ & $\begin{array}{c}-0.0675 \pm 0.0057 \\
-0.0125 \pm 0.0021 \\
0.0038 \pm 0.0014 \\
0.0013 \pm 0.0016\end{array}$ \\
\hline Chip 4 & $\begin{array}{l}U \\
B \\
V \\
I\end{array}$ & $\begin{array}{r}0.0208 \pm 0.0020 \\
0.0195 \pm 0.0007 \\
-0.0060 \pm 0.0005 \\
-0.0055 \pm 0.0005\end{array}$ & $\begin{array}{c}-0.0156 \pm 0.0062 \\
-0.0093 \pm 0.0021 \\
0.0016 \pm 0.0015 \\
0.0026 \pm 0.0016\end{array}$ \\
\hline Chip 5 & $\begin{array}{l}U \\
B \\
V \\
I\end{array}$ & $\begin{array}{c}0.0409 \pm 0.0020 \\
-0.0024 \pm 0.0007 \\
-0.0149 \pm 0.0005 \\
0.0019 \pm 0.0007\end{array}$ & $\begin{array}{c}0.0013 \pm 0.0057 \\
-0.0018 \pm 0.0022 \\
0.0022 \pm 0.0016 \\
-0.0006 \pm 0.0014\end{array}$ \\
\hline Chip 7 & $\begin{array}{l}U \\
B \\
V \\
I\end{array}$ & $\begin{array}{c}-0.0183 \pm 0.0020 \\
-0.0052 \pm 0.0007 \\
-0.0023 \pm 0.0004 \\
0.0031 \pm 0.0005\end{array}$ & $\begin{array}{c}-0.0016 \pm 0.0060 \\
0.0047 \pm 0.0021 \\
-0.0002 \pm 0.0014 \\
0.0033 \pm 0.0017\end{array}$ \\
\hline Chip & $\begin{array}{l}U \\
B \\
V \\
I\end{array}$ & $\begin{array}{c}0.0136 \pm 0.0020 \\
0.0083 \pm 0.0007 \\
-0.0046 \pm 0.0005 \\
0.0122 \pm 0.0005\end{array}$ & $\begin{array}{c}-0.0069 \pm 0.0060 \\
-0.0037 \pm 0.0021 \\
0.0005 \pm 0.0015 \\
0.0035 \pm 0.0017\end{array}$ \\
\hline
\end{tabular}

the color coefficients. The colors used in the calibration are $U-B, B-V, B-V$, and $V-I$ for $U, B, V$, and $I$, respectively. The photometric coefficients are determined by an iterative process of error-weighted fitting of the transformation equation to the standard magnitudes. The quadratic color term is included only in the equation of $U$ band, because a significant deviation from a linear fit occurs in the transformation of $U$ band. Figure 2 shows the $U$ magnitude difference between the standard and instrumental magnitudes corrected for both of the photometric zero point and the atmospheric extinction, as a function of $U-B$. The dashed lines represent two best-fit functions for blue and red standard stars, respec- tively. However, if we divide the observed data into two groups by $U-B$ and use the two functions in calibration separately, the discontinuity between two functions may lead to an artificial clump in color distributions of the data. To avoid this problem, we connected the two functions (red lines) at the internal division points of them in the range $0.29<U-B<1.35$ (two vertical lines). Finally, the instrumental magnitudes were converted to the standard Johnson-Cousins $U B V I$ system using the set of photometric coefficients listed in Table 3

Calibrating the SDSS $u$ photometry to the Johnson $U$ system may cause systematic shifts in the colors based on this band since the system throughputs are different between the two filters. To estimate these shifts, we simulated our observations using the system throughputs of the two filters, synthetic spectra of GCs from the YEPS model (Chung et al. 2012), and stars from the Kurucz ATLAS9 model. We calculated the magnitude through each filter for each model GC, and converted it to the standardized Johnson $U$ magnitude using the synthetic spectrum of a star having the same color of the GC. We then calculated the differences between these standardized magnitudes from the respective filters. The differences range from $0.002 \mathrm{mag}$ to $0.022 \mathrm{mag}$, depending on the colors of the model GCs. We conclude that these are small enough to have negligible impact on our photometric analyses throughout the paper.

As the individual CCD chips of the Mosaic may have different color terms, we attempted to correct for this "chip-to-chip variation" as follows. On the night of 2008 May 5, we took multiple $u B V I$ observations of the Landolt standard fields SA98 and SA107 with the Mosaic II, such that the same set of standard stars was positioned on each chip. Using these data, we derived the color terms that correct for the chip-to-chip variation. Although these color terms were very small, we applied them to our data in hopes of making the multiple measurements of the same source more consistent. The equation used in correction is of the form

$$
M_{\text {cor }}=m+\mathrm{c} 1+\mathrm{c} 2 \times \text { color, }
$$

where $M_{\text {cor }}$ is the magnitude converted to that on the reference chip $6, m$ is the magnitude measured on each chip, c1 is the zero point offset, and c2 is the color coefficient. The colors used in the calibration are $U-B$, $B-V, B-V$, and $V-I$ for $U, B, V$, and $I$, respectively. Table 4 lists the zero point offsets and color coefficients 


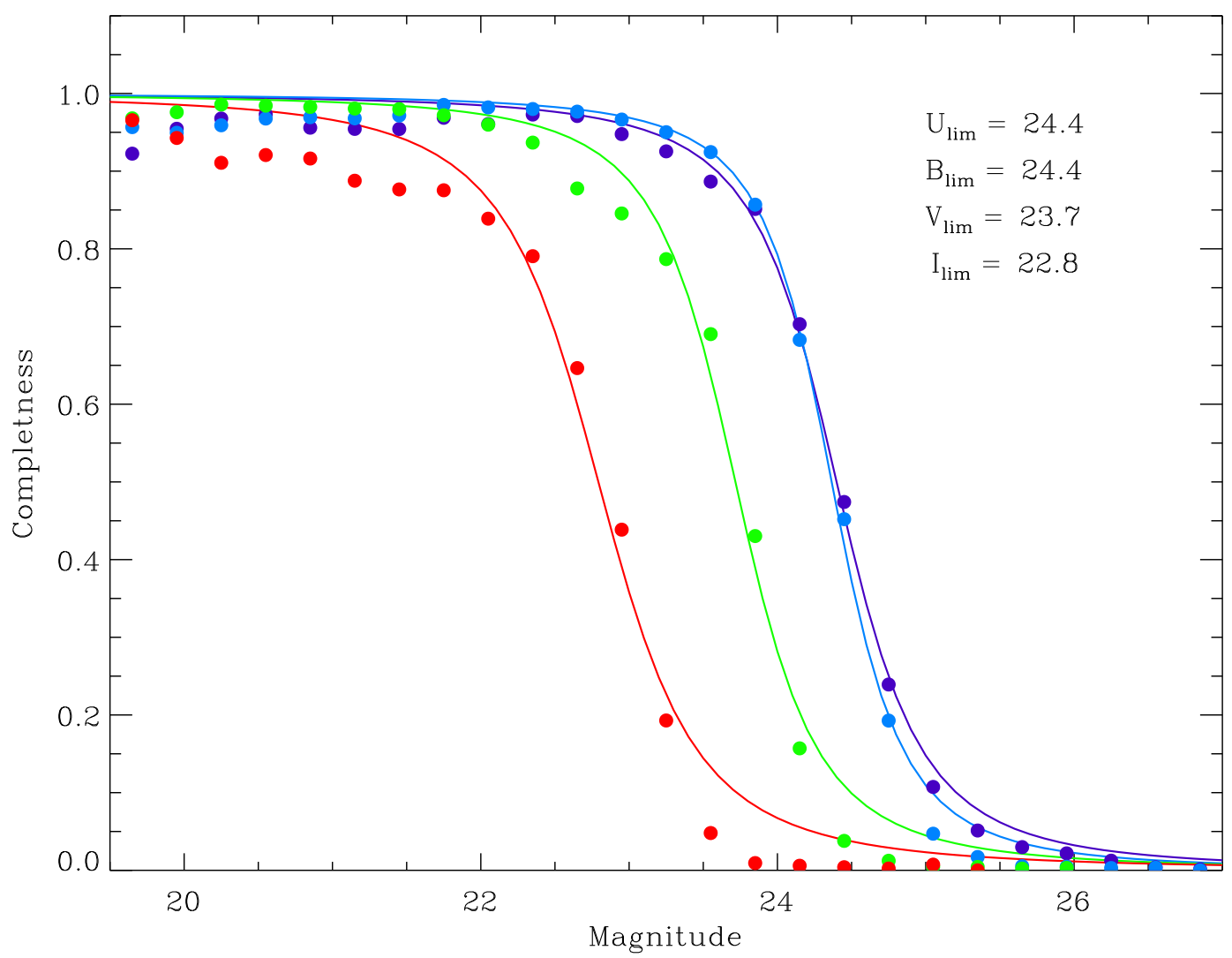

FIG. 3.- Completeness functions obtained from artificial star tests. Detection efficiencies (filled circle) were fitted to the analytic function by Fleming et al. (1995), which are denoted by solid lines. The purple, blue, green, and red lines represent the $U, B, V$, and $I$ bands, respectively. The functions are for the overall image, except the galactic central regions and the gaps between mosaic chips where the detection efficiency rapidly drops. Limiting magnitudes where the completeness drops to $50 \%$ are indicated in the upper-right corner.

for each chip.

We corrected for the foreground Galactic extinction using the reddening maps provided by Schlegel et al. (1998). To account for the atmospheric dispersion corrector (ADC) of the $4 \mathrm{~m}$ Blanco telescope that significantly modifies the system throughputs, we computed the effective wavelengths of our four bands from the total system throughputs including the effect of the ADC, and we derived $A_{\lambda} / E(B-V)$ values based on the extinction law provided by Cardelli et al. (1989). The mean extinction values in $U, B, V$, and $I$ are $0.06,0.05,0.04$, and 0.02 mag, respectively.

Our images were calibrated for astrometry using the USNO-B 1.0 catalog stars (Monet et al. 2003) and the MSCTPEAK task. The typical rms of our astrometric solutions is at the level of $0^{\prime \prime} .2$. The R.A./decl. coordinates of our subsequent source catalogs use the positions derived from these images.

\subsection{Completeness}

We performed artificial star tests to estimate the completeness of our photometry. We used the ADDSTAR routine of DAOPHOT II to generate 4000 artificial stars in each chip with magnitudes and color ranges consistent with those of the point-sources detected and measured above. The luminosity function of our artificial stars was chosen to be flat to preserve statistical significance in all magnitude ranges. Artificial stars were added to the PSF-subtracted images using the empirical PSFs derived during our photometry. We used the same photometry procedure as above to detect and measure artificial stars in these images. This process was repeated seven times with the positions of artificial stars altering randomly each time, resulting in a total of 28,000 artificial stars per chip. In each 0.3 mag bin, we calculated the detection efficiency, i.e., the ratio of the number of recovered artificial stars to the number of added ones. Central regions of galaxies and the gaps between CCD chips were masked for the calculation. To obtain a limiting magnitude at which the completeness drops to $50 \%$, the detection efficiencies were fitted to the analytic function by Fleming et al. (1995),

$$
f(m)=\frac{1}{2}\left(1-\frac{\alpha\left(m-m_{\mathrm{lim}}\right)}{\sqrt{1+\alpha^{2}\left(m-m_{\mathrm{lim}}\right)^{2}}}\right),
$$

where $m_{\lim }$ is the limiting magnitude, and $\alpha$ is the parameter that controls the steepness of the completeness function near the limiting magnitude. Figure 3 shows the measured completeness (dots) and the fitted functions (solid lines) in $U, B, V$, and $I$ bands depicted by purple, blue, green, and red colors, respectively. The limiting magnitudes of the four bands are 24.4, 24.4, 23.7, and $22.8 \mathrm{mag}$ in $U, B, V$, and $I$ bands, respectively. We note that the limiting magnitudes slightly vary in the $<0.2$ mag level among individual CCDs due to variations in 

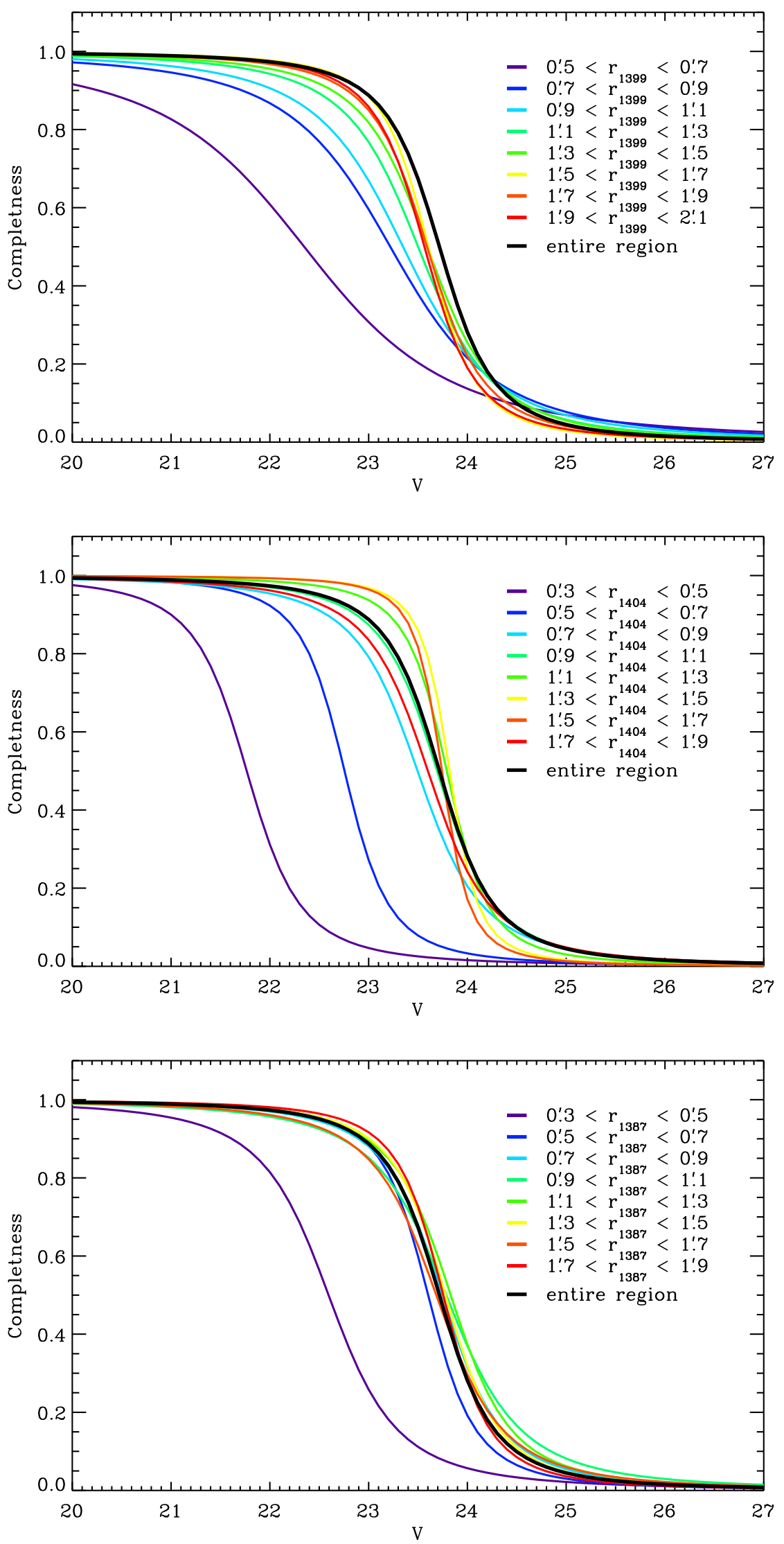

FIG. 4.- $V$-band completeness functions at various radial bins from the center of galaxies - NGC 1399 (top), NGC 1404 (middle), and NGC 1387 (bottom). The black thick line represents the overall completeness function. The completeness is very low near the center of the galaxies, and increases as one moves out from the center, and then flattens out beyond 1!3 for NGC 1399, 0!7 for NGC 1404, and 0!5 for NGC 1387. 


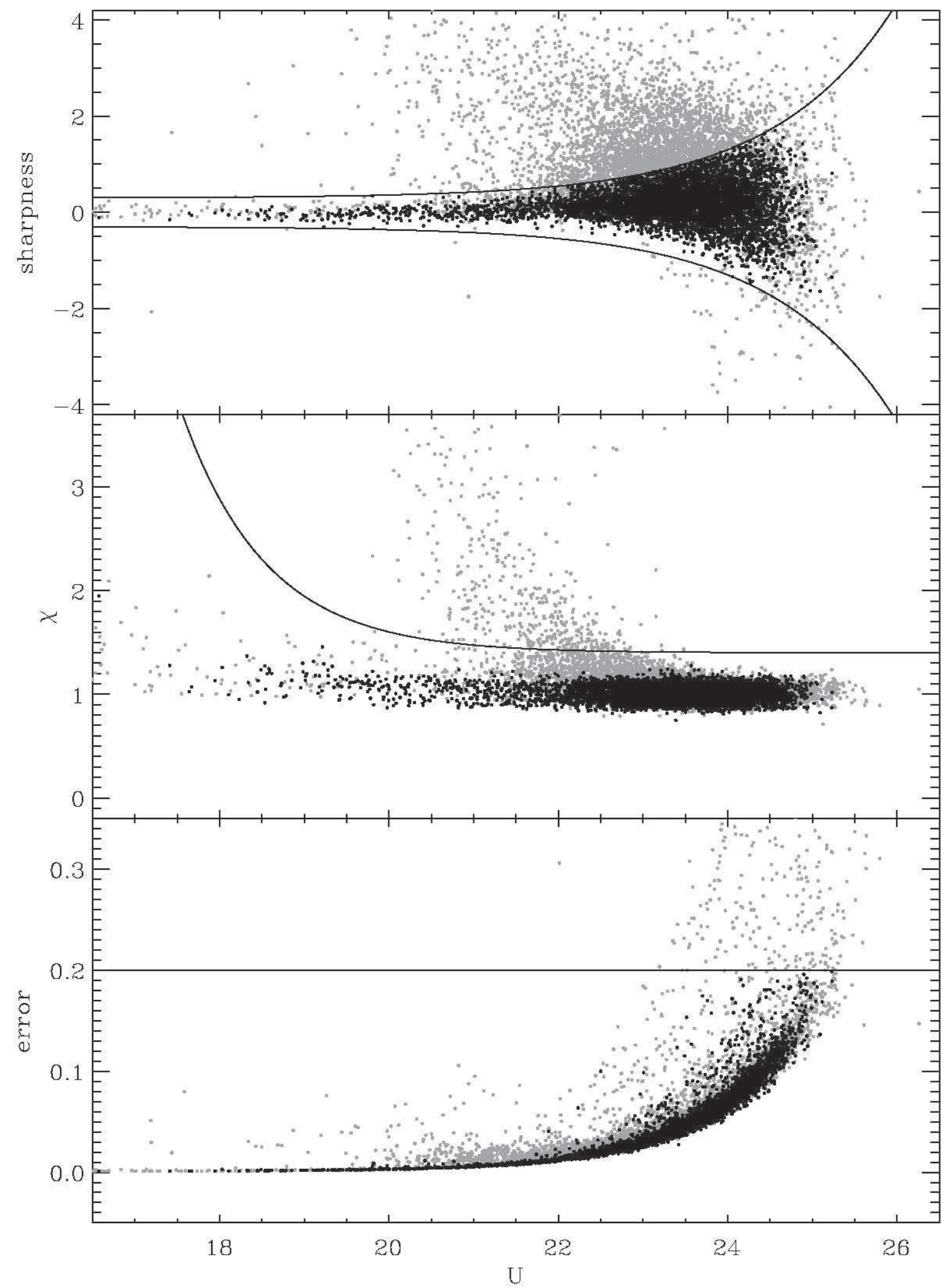

FIG. 5.- Photometric error and image parameters ( $\chi$ and Sharpness) in the $U$ band as a function of magnitude. Solid lines show the point-source selection criteria. Black dots represent the selected sources with good photometry, while gray dots represent the sources that do not satisfy any of the selection criteria. 
detector characteristics.

We also investigated the radial variations of completeness around NGC 1399, NGC 1404, and NGC 1387. In the central region of a galaxy, the detection effectiveness at a certain magnitude decreases as the surface brightness of the galaxy increases, and it finally becomes zero at a saturated region. To quantify this effect in our observations, we performed separate artificial star tests near the galaxy centers. We added 8000 artificial stars on the individual CCD chips that contain the galaxies (NGC 1399, NGC 1404, and NGC 1387), this time with a centrally concentrated radial distribution centered on each galaxy. This experiment was repeated five times with the positions of artificial stars altering each time. The completeness was then calculated as a function of magnitude in concentric circles with radial intervals of 0.2 . Figure 4 shows the $V$-band completeness functions at various radial bins from the center of each galaxy. The thick black line represents the completeness function for the overall region, as derived above. As expected from the strong background lights near the centers of our target galaxies, we find that the completeness is very low near the center. The completeness increases as one moves out from the center, and then flattens out beyond 1'.3 for NGC 1399, 0.7 for NGC 1404, and 0.5 for NGC 1387.

\section{CONSTRUCTION OF GLOBULAR CLUSTER CATALOGS}

\subsection{Selection of Globular Cluster Candidates}

At the distance of the Fornax cluster $(\sim 20.0 \mathrm{Mpc}$; Blakeslee et al. 2009), 1 pc is equal to 0.'01, so a typical GC appears as a point-source in our Mosaic images. We therefore can not select GCs based on their apparent sizes but must rely on photometric colors. Details on the selection process of GC candidates are given below.

We start by using the ALLFRAME parameters $\mathrm{CHI}$ and SHARP to select point-sources from our photometric catalog. Figure 5 shows the SHARP, CHI, and the ALLFRAME photometric error as a function of $U_{0}$ magnitude with solid lines showing the selection criteria. While the figure only shows the $U$-band selection, we applied the same selection criteria to all bands simultaneously, and the resulting selection includes 5500 point-source objects. We then employ color cuts using our multi band photometry as demonstrated in Figure 6 to select GC candidates. The blue selection boxes shown in the three color-color diagrams were determined on the basis of the findings below. First, we matched our point-source objects with the spectroscopic catalog of Schuberth et al. (2010) and found that the NGC 1399 GCs, as identified by their measured line-of-sight velocities, form a tight sequence (red dots in Figure 6) in the color-color diagrams, whereas foreground stars are located along an extended sequence (cyan dots in Figure6). Second, we downloaded Hubble Space Telescope (HST)/ACS images of NGC 1399 from the archive and identified genuine GCs based on their sizes. The sizes were measured using ISHAPE (Larsen 1999) and following the same procedure outlined in Strader et al. (2006). We then cross-identified the GCs with our point-source objects, and found that most of them lie inside the tight sequence (orange dots in Figure 6). To select GC candidates, we further applied a magnitude cut of $V_{0} \leq 20.6$ (corresponding to $\left.M_{V} \lesssim-11\right)$ to the color-color-selected sample. This
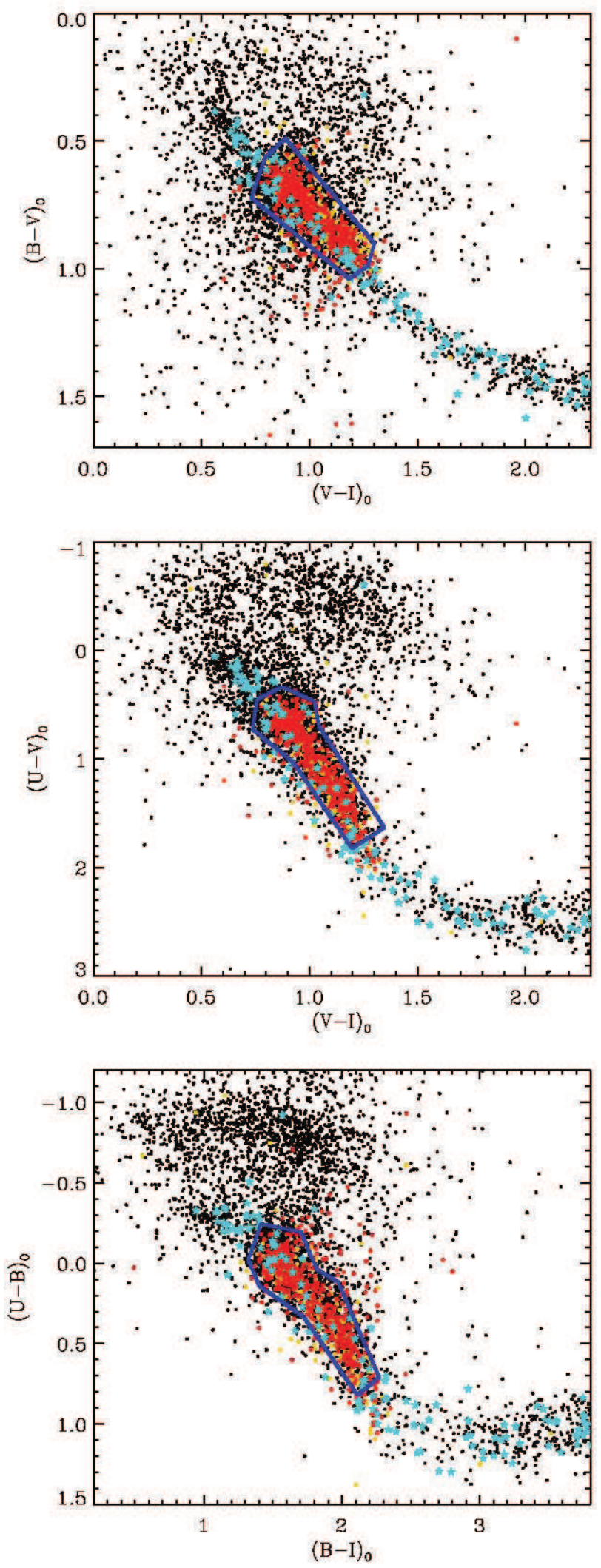

FIG. 6.- Color selection of GC candidates in NGC 1399. The selection criteria demarcated by blue lines were determined based on confirmed GCs with radial velocity measurements (red dots; Schuberth et al. 2010) and/or with size measurements (orange dots; derived from HST ACS data). The cyan asterisks represent foreground stars confirmed with radial velocity measurements Schuberth et al. 2010). GC candidates are clearly separated from background sources in the $(U-B)_{0}$ color. 
magnitude limit was determined based on the brightest GC in the spectroscopic catalog of Schuberth et al. (2010), and we note that it is consistent with the limit used by Mieske et al. (2006) to separate GCs from their ultra compact dwarf galaxies sample. The color-color and magnitude cuts result in a final sample of 2037 GC candidates.

Figure 6 demonstrates the advantage of using $U$ band for separating star-forming background galaxies from the extragalactic GCs. The background galaxies have ( $B-$ $V)_{0}$ colors similar to the bluer GCs, but due to their starforming nature, galaxies exhibit significantly bluer colors in $(U-V)_{0}$ and $(U-B)_{0}$ than the GCs. This allows us to effectively reduce contamination by background galaxies. The same effect was found by Dirsch et al. (2003) using the $C-T 1$ Washington color index. Nonetheless, our selection box still contains several foreground stars, and it is important to quantify the contamination level of our color-color-selected objects. We provide the estimation of the contamination level below.

\subsection{Estimation of Contamination}

To estimate the number of contaminating sources (foreground stars and background galaxies) in our GC candidate list, it would be best to use a control field observed near the science field with the same observational conditions. However, this approach is impractical for us because the $U$-band observations require very long exposure times. As an alternative, we used two independent methods to estimate the contamination level of our $\mathrm{GC}$ candidate list.

On average, chip 1 is located farthest away from the target galaxies NGC 1399, NGC 1387, and NGC 1404, so it has the least number of GCs expected among the eight CCD chips In Figure 7. we compare the $(U-$ $B)_{0}$ versus $(B-I)_{0}$ color-color diagrams of the 5500 point-source objects for each chip. In chip 1 , there are 41 objects that are selected as GC candidates using the color-color selection box as in Figure 6] If we assume that all 41 objects are contaminants, this implies that our catalog of GC candidates has a contamination level of $16 \%$. We note that $16 \%$ is in fact an upper limit because chip 1 , which is at an average distance of $24^{\prime}(\sim 140 \mathrm{kpc})$ from the center of NGC 1399, is likely to contain several GCs given that the NGC 1399 GCs are known to extend out to a projected distance of at least $45^{\prime}(\sim 260 \mathrm{kpc}$; Bassino et al. 2006a).

We further estimated the number of foreground stars using the Besançon model of the Milky Way population (Robin et al. 2003). We generated a synthetic catalog of stars in the direction of the sky consistent with our target field and then selected stars using the same colorcolor cut as used in Figure 6. The estimated number of foreground stars in our GC candidate list turned out to be 180 and the corresponding contamination level is $9 \%$. As expected, this is lower than the upper limit set by using chip 1 as the control field above. Based on the two estimates, the contamination level of our GC candidate list is likely to be in the $9 \%-16 \%$ range.

Although the contamination level itself may not be that high, it is important to test whether the contaminat-

\footnotetext{
2 NGC 1389 is known to have very few GCs (see Section 4.1), so we only considered the other three Fornax galaxies here.
}

ing sources will affect our GC color distribution analyses. Figure 8 shows the comparison of $(U-B)_{0}$ color distributions for all point-sources (shaded histogram), our GC candidate list (blue), and synthetic stars from the Besançon model (red) between chips 7 and 1. Based on the similarities found in the red and blue color distributions in the upper panel of Figure 8, chip 1 seems to be a good control field. From the same figure, we find that the foreground MW stars are expected to have a $(U-B)_{0}$ color distributions that is peaked toward the bluer side similar to the $(U-B)_{0}$ color distribution of GC candidates. However, as demonstrated in the lower panel of Figure 8, the number of these foreground stars, when compared to that of the GC candidates, is too small to have any impact on our color distribution analyses presented in Sections 4.3 and 4.4.

\subsection{Photometric Catalog}

Table 5 presents the photometric catalog of total 2,037 $\mathrm{GC}$ candidates. The columns give the following information.

Column 1. Identification number

Columns 2 and 3. Right ascension and declination (J2000)

Columns 4 and 5. $U_{0}$ magnitude and its rms uncertainty Columns 6 and \%. $B_{0}$ magnitude and its rms uncertainty Columns 8 and 9. $V_{0}$ magnitude and its rms uncertainty Columns 10 and 11. $I_{0}$ magnitude and its rms uncertainty.

We note that all magnitudes in the catalog are corrected for Galactic extinction.

\section{RESULTS AND DISCUSSION}

\subsection{Spatial Distribution}

Figure 9 maps the spatial distributions of GC candidates (left panel) and foreground/background objects (right). The color-filled contours show the distribution of surface number density. The black crosses mark the positions of the optical centers of galaxies (from top, NGC 1399, NGC 1387, NGC 1404, and NGC 1389). In the left panel, three GC systems are clearly visible around NGC 1399, NGC 1404, and NGC 1387. The number of GCs around NGC 1389 is too small, so we omit the galaxy from the following analysis. In the right panel, the distribution of foreground/background objects appears fairly uniform. The slight overdensity in the region between NGC 1399 and NGC 1387 is likely due to the presence of a distant galaxy cluster, given that many objects in this region are bluer than -0.3 in $(U-B)_{0}$. Another overdense region is right on the center of NGC 1399, which indicates that GCs in highly dense regions can be misclassified as foreground/background objects in our GC selection procedure, as mentioned in Section 3. Note that the residuals of the mosaic CCD gaps are discernible as a vertical feature in the middle of the panel.

It is noteworthy that the GC distribution around NGC 1399 shows interesting features. On the one hand, there is an overabundance of GCs in the outer region of NGC 1399 toward NGC 1404 and NGC 1387. This was also pointed out by Bassino et al. (2006a), and it may indicate interactions of NGC 1399 with NGC 1404 and NGC 1387 in the recent past (Forbes et al. 1997; Bekki et al. $2003)$. On the other hand, a $\sim 0.5$ displacement is found 


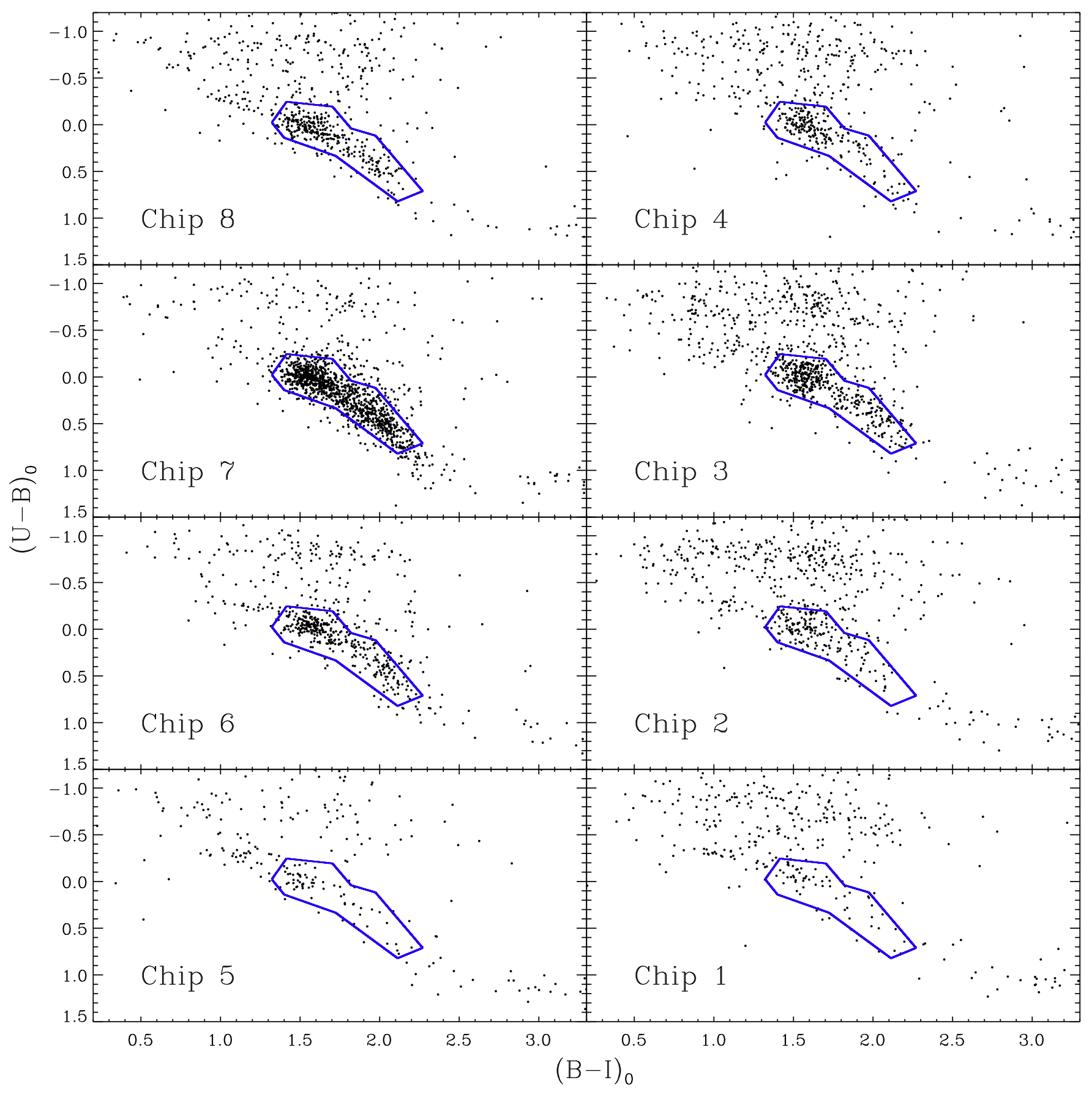

FIG. 7.- Color-color diagrams of point-sources on each chip. Solid boxes indicate the selection criteria for the GC candidates and the chip numbers are indicated in the lower-left corner of each panel. Chips 7, 6, and 3 contain NGC 1399, NGC 1404, and NGC 1387, respectively. Chip 1, which is located farthest away from the galaxies, is used to estimate the upper limit of the contamination level of our data.

between NGC 1399's optical center and the center of the GC distribution in the inner region of the galaxy as denoted by a red contour in the density map. Paolillo et al. (2002) reported an asymmetric X-ray halo for NGC 1399, consisting of three components with different centers; a central component, a galactic component centered $1^{\prime}$ southwest of NGC 1399, and a cluster component centered $\sim 5 ! 6$ northeast of the galaxy. While the center of the $\mathrm{GC}$ distribution does not coincide with the X-ray centers, the asymmetric distribution of the GCs may sug- gest that the NGC 1399 is not yet dynamically relaxed and may be undergoing merger events.

Figure 10 presents the surface number densities against the galactocentric radius for the three galaxies. In order to examine the radial extent of each GC system, we calculated the surface GC number density as a function of the distance from the galaxy center. We first set up a series of radial bins (annulus), each containing approximately equal numbers of GCs ( 100 GCs for NGC 1399; $\sim 40$ GCs for NGC 1404; 25 GCs for NGC 1387). The 


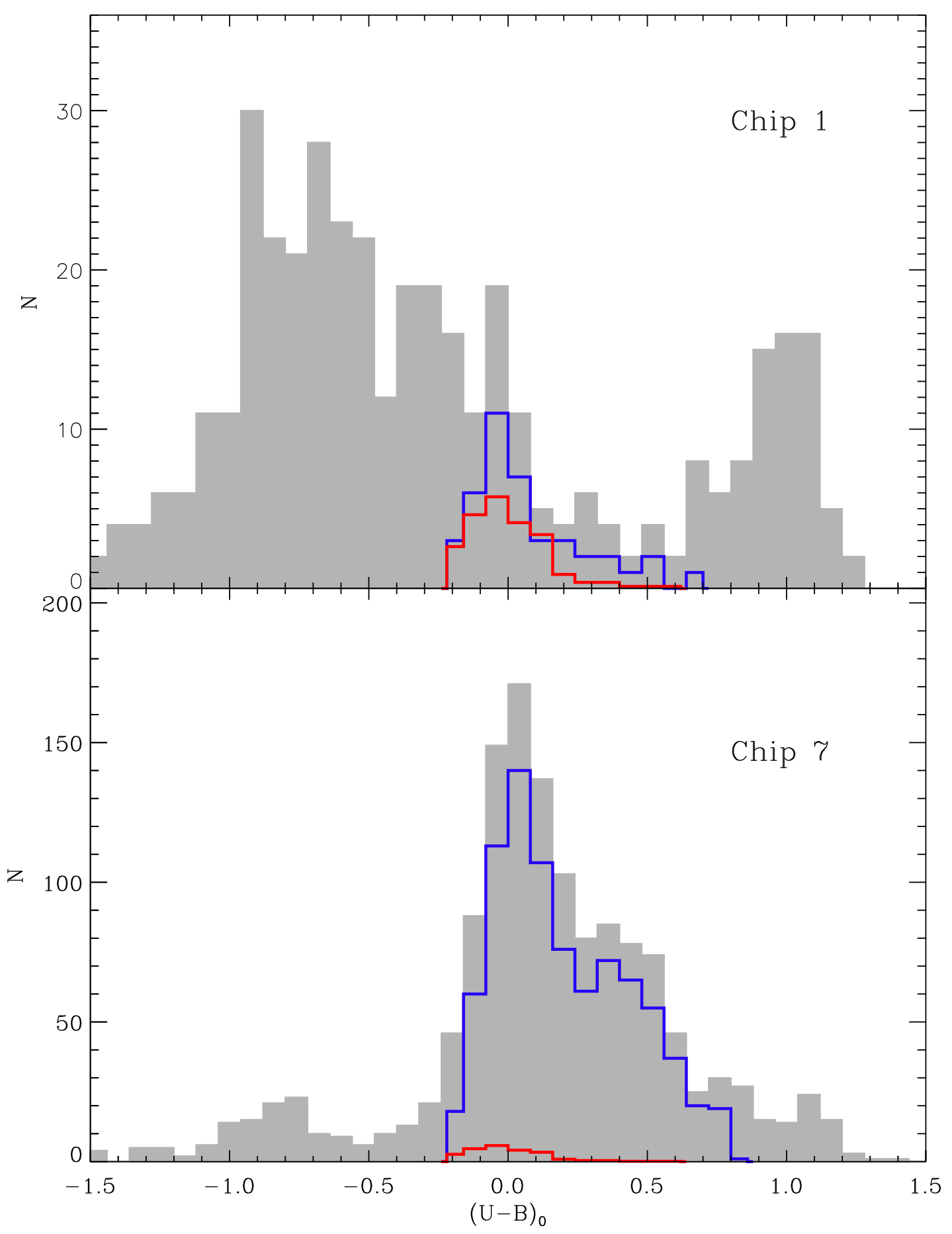

FIG. 8.- $U-B$ color distributions for all point-sources (shaded histogram), GC candidates (blue), and synthetic stars from Besançon model (red) in chip 1 and chip 7. The similarities found in the red and blue color distributions in the upper panel indicate that chip 1 is a good control field. 

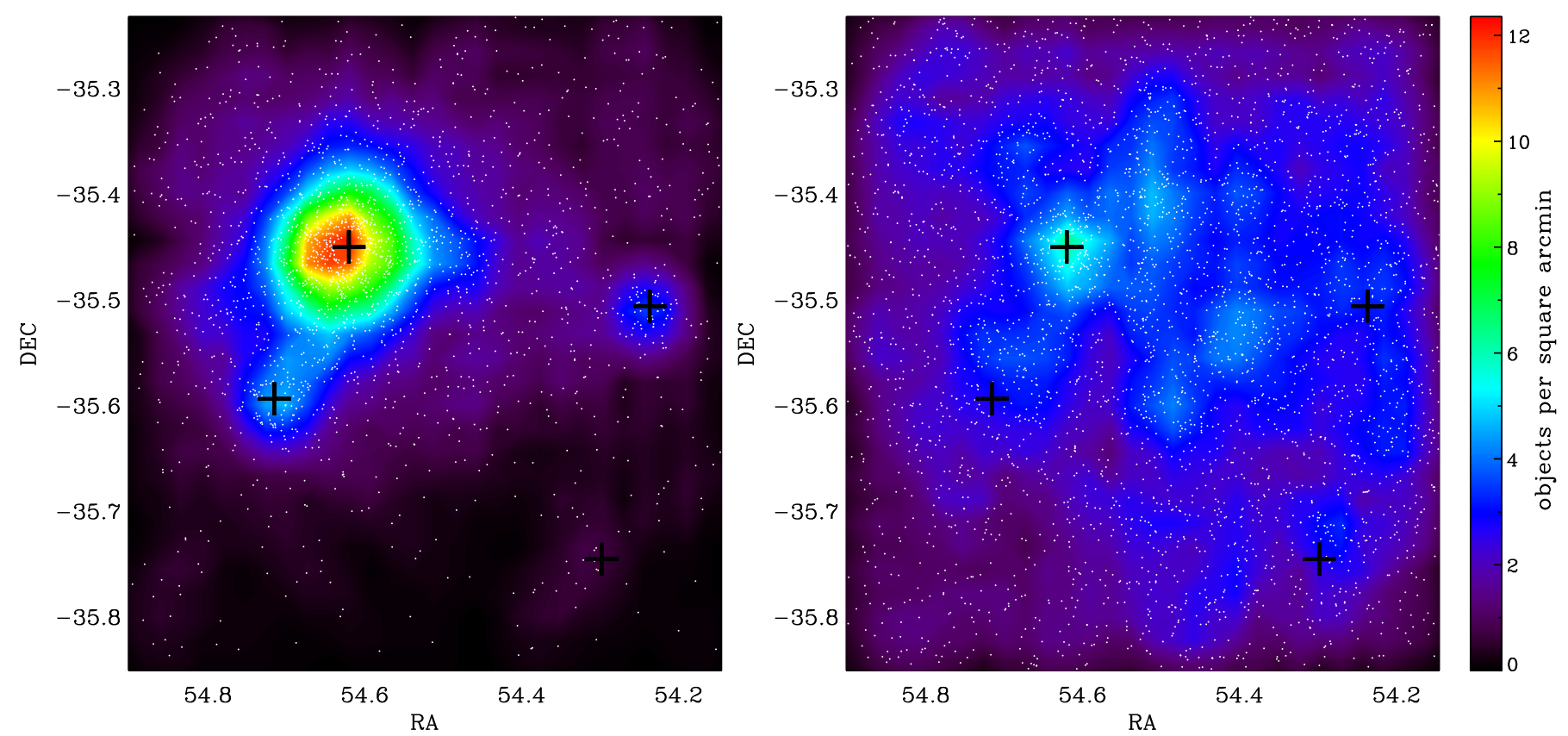

FIG. 9. - Spatial distribution of GC candidates (left) and foreground/background objects (right) over color-filled density contour maps with the color scale from black to red for increasing surface number density. Black crosses represent the positions of the optical centers of galaxies (from top, NGC 1399, NGC 1387, NGC 1404, and NGC 1389).

TABLE 5

U $B$ I Photometry of GC Candidates in the Fornax Galaxy Cluster

\begin{tabular}{ccccccccccc}
\hline \hline GC ID & R.A. (J2000) & Decl. (J2000) & $U_{0}$ & $U$ Error & $B_{0}$ & $B$ Error & $V_{0}$ & $V$ Error & $I_{0}$ & $I$ Error \\
\hline 1 & 54.4670781 & -35.8412594 & 23.863 & 0.057 & 23.466 & 0.018 & 22.611 & 0.025 & 21.614 & 0.018 \\
2 & 54.4048663 & -35.8368808 & 23.099 & 0.028 & 22.941 & 0.011 & 22.069 & 0.015 & 21.119 & 0.010 \\
3 & 54.1532595 & -35.8313662 & 23.048 & 0.038 & 22.743 & 0.012 & 21.970 & 0.015 & 20.920 & 0.020 \\
4 & 54.3887923 & -35.8307234 & 24.085 & 0.070 & 23.983 & 0.029 & 23.384 & 0.049 & 22.462 & 0.030 \\
5 & 54.3871824 & -35.8300091 & 23.772 & 0.054 & 23.865 & 0.025 & 23.147 & 0.038 & 22.327 & 0.028 \\
6 & 54.3545131 & -35.8284302 & 23.147 & 0.030 & 22.633 & 0.008 & 21.661 & 0.011 & 20.490 & 0.006 \\
7 & 54.5147090 & -35.8246663 & 22.822 & 0.023 & 22.949 & 0.014 & 22.247 & 0.015 & 21.430 & 0.013 \\
8 & 54.3057038 & -35.8223111 & 24.007 & 0.061 & 24.027 & 0.028 & 23.363 & 0.045 & 22.429 & 0.028 \\
9 & 54.2008513 & -35.8137756 & 24.064 & 0.070 & 24.111 & 0.030 & 23.383 & 0.045 & 22.486 & 0.030 \\
10 & 54.4062928 & -35.8090401 & 21.291 & 0.007 & 21.338 & 0.004 & 20.674 & 0.005 & 19.854 & 0.003 \\
$\cdots$ & $\cdots$ & $\cdots$ & $\cdots$ & $\cdots$ & $\cdots$ & $\cdots$ & $\cdots$ & $\cdots$ & $\cdots$ & $\cdots$ \\
$\cdots$ & $\cdots$ & $\cdots$ & $\cdots$ & $\cdots$ & $\cdots$ & $\cdots$ & $\cdots$ & $\cdots$ & $\cdots$ & $\cdots$ \\
$\cdots$ & $\cdots$ & $\cdots$ & $\cdots$ & $\cdots$ & $\cdots$ & $\cdots$ & $\cdots$ & $\cdots$ & $\cdots$ & $\cdots$ \\
\hline
\end{tabular}

Note. The first 10 rows of the table are reproduced here; the full version of this table containing the 2,037 GC candidates is available online at the CDS. 


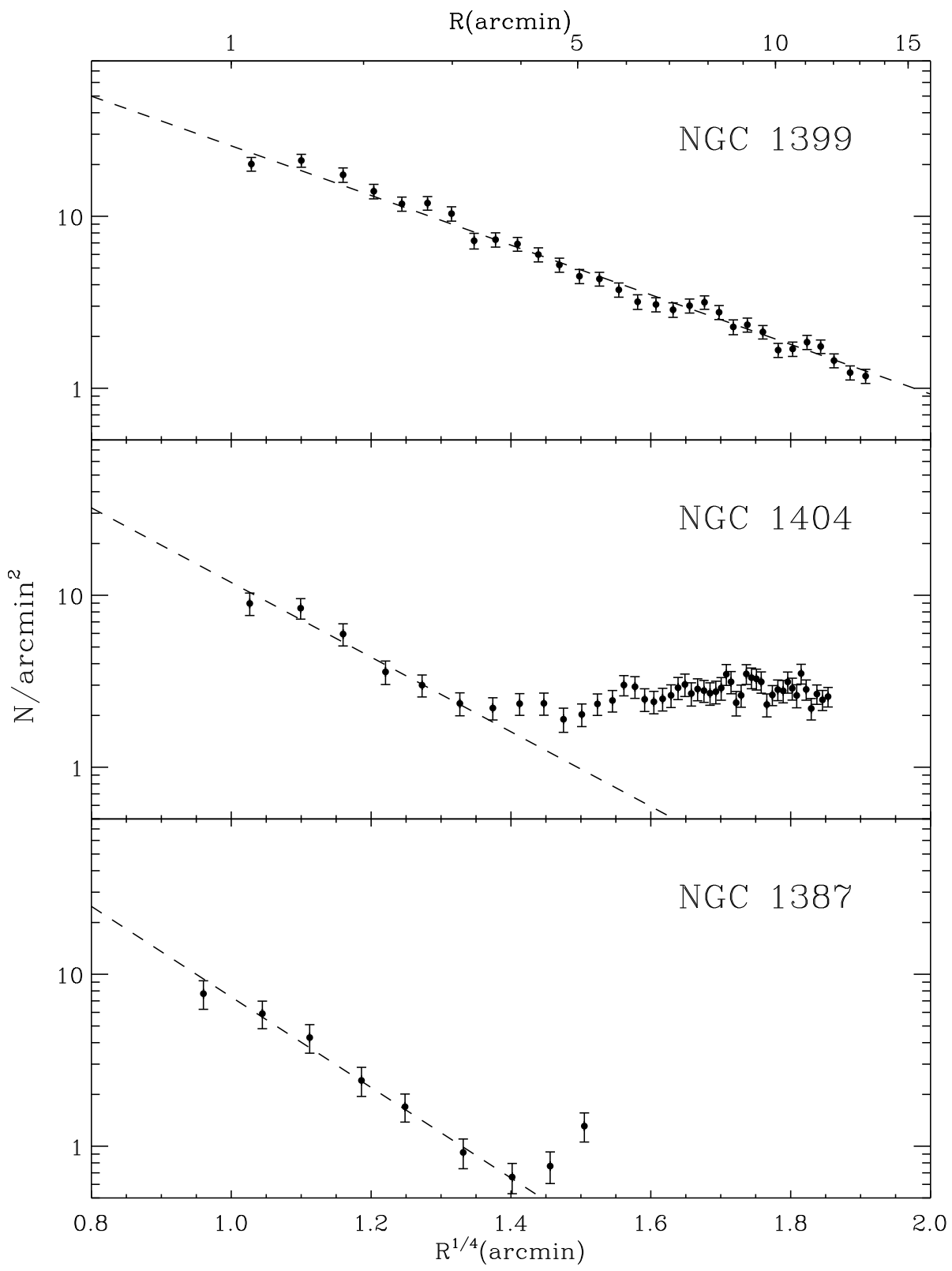

FIG. 10. - Number of GC candidates per unit area projected on the sky as a function of radial distance from the center of the galaxy. Radial bins are defined to contain approximately equal numbers of GCs $(\sim 100$ GCs for NGC $1399 ; \sim 40$ GCs for NGC 1404; $\sim 25$ GCs for NGC 1387). The numbers are corrected for the spatial completeness, but not for the contamination by foreground/background objects (see the text). Dashed line shows de Vaucouleurs' $R^{1 / 4}$ fit of each profile.

number of GCs in each bin was then corrected using the corresponding completeness function of the bin. We finally divided the corrected numbers of GCs by the area of annuli to get surface densities. The surface density profile of each galaxy is well described by de Vaucouleurs' $R^{1 / 4}$ fits (dashed lines). We determine the limiting radii of GC systems of NGC $1404(3 ! 4)$ and NGC $1387(3 ! 7)$ as the radial points where the density profiles begin to depart from de Vaucouleurs' law. Since the GC system of NGC 1399 is extended ( $\sim 45^{\prime}$; Bassino et al. 2006a) well beyond the field of view $\left(36^{\prime} \times 36^{\prime}\right)$ of our Mosaic observations, we regard the entire GCs in the images as NGC 1399's GC system, except for the regions inside the limiting radii of NGC 1404 and NGC 1387.
Figure 11 presents the CMDs for all point-sources. Magnitudes and colors were corrected for the Galactic extinction, as mentioned in Section 2.3. The black and gray dots represent the GC candidates and foreground/background objects, respectively. The dashed lines indicate the limiting magnitude of $50 \%$ completeness. The tilt of the limiting magnitude lines results from lower detection efficiency of red GCs than blue GCs in the $U$ band. The $(U-B)_{0}$ colors corresponding to GCs, background galaxies, and foreground stars can be determined both by the $(U-B)_{0}$ versus $(B-I)_{0}$ diagram (the bottom panel of Figure 6$)$ and by the $V_{0}-(U-B)_{0}$ CMD (the left panel of Figure 11). GCs are placed at $-0.2<(U-B)_{0}<0.8$, while most background galaxies 

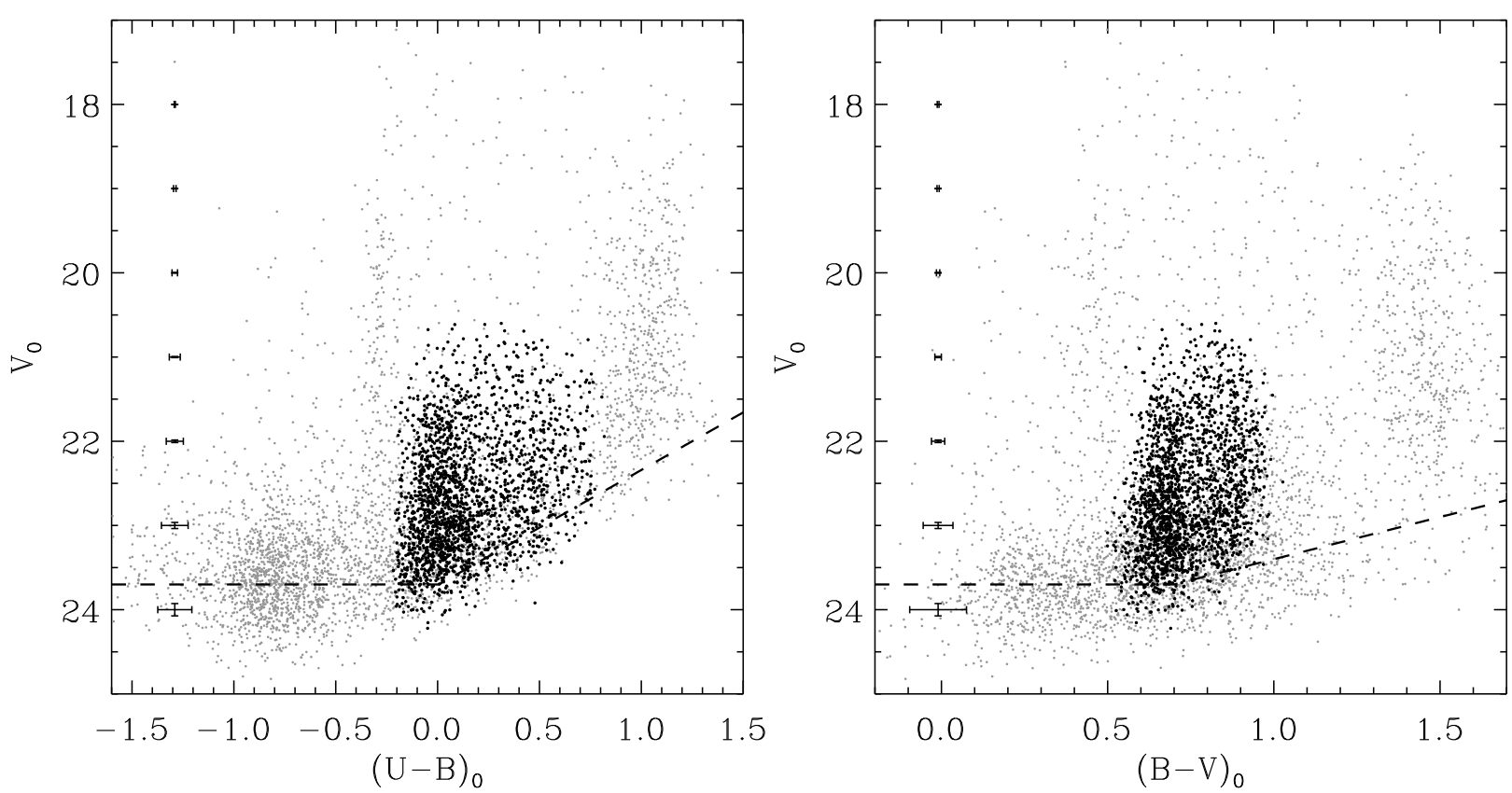

FIG. 11. - Color-magnitude diagrams of point-sources. Black dots represent GC candidates, and gray dots represent foreground/background objects. Observational uncertainties as a function of $V_{0}$ are shown by error bars in the left corner of each diagram. Dashed lines indicate the limiting magnitude. The tilt of the limiting magnitude results from lower detection efficiency of red GCs than blue GCs in the $U$ band. The GC candidates fainter than the limiting magnitude are taken out from the subsequent analysis.

TABLE 6

Results from GMM Analysis for Color Distributions of GCs

\begin{tabular}{|c|c|c|c|c|c|c|c|c|c|}
\hline Color & $\mu_{b}$ & $\sigma_{b}$ & $\mu_{r}$ & $\sigma_{r}$ & $f_{r}$ & $\sigma_{r} / \sigma_{b}$ & $p\left(\chi^{2}\right)$ & $p(D D)$ & $p(k u r t)$ \\
\hline \multicolumn{10}{|c|}{ NGC 1399} \\
\hline $\begin{array}{l}U-B \\
U-V \\
U-I \\
B-V \\
B-I \\
V-I\end{array}$ & $\begin{array}{l}0.016 \pm 0.006 \\
0.670 \pm 0.007 \\
1.557 \pm 0.008 \\
0.673 \pm 0.004 \\
1.571 \pm 0.005 \\
0.910 \pm 0.005\end{array}$ & $\begin{array}{l}0.102 \pm 0.003 \\
0.113 \pm 0.005 \\
0.134 \pm 0.006 \\
0.058 \pm 0.002 \\
0.087 \pm 0.003 \\
0.063 \pm 0.003\end{array}$ & $\begin{array}{l}0.372 \pm 0.021 \\
1.147 \pm 0.022 \\
2.188 \pm 0.025 \\
0.849 \pm 0.007 \\
1.925 \pm 0.014 \\
1.108 \pm 0.013\end{array}$ & $\begin{array}{l}0.188 \pm 0.009 \\
0.269 \pm 0.008 \\
0.357 \pm 0.008 \\
0.060 \pm 0.003 \\
0.135 \pm 0.007 \\
0.068 \pm 0.007\end{array}$ & $\begin{array}{l}0.34 \\
0.42 \\
0.45 \\
0.34 \\
0.37 \\
0.30\end{array}$ & $\begin{array}{l}1.84 \\
2.38 \\
2.66 \\
1.03 \\
1.55 \\
1.08\end{array}$ & $\begin{array}{l}0.010 \\
0.010 \\
0.010 \\
0.010 \\
0.010 \\
0.010\end{array}$ & $\begin{array}{l}0.090 \\
0.100 \\
0.080 \\
0.070 \\
0.050 \\
0.060\end{array}$ & $\begin{array}{l}0.860 \\
0.140 \\
0.010 \\
0.010 \\
0.010 \\
0.010\end{array}$ \\
\hline \multicolumn{10}{|c|}{ NGC1404 } \\
\hline $\begin{array}{l}U-B \\
U-V \\
U-I \\
B-V \\
B-I \\
V-I\end{array}$ & $\begin{array}{c}-0.004 \pm 0.012 \\
0.682 \pm 0.015 \\
1.631 \pm 0.018 \\
0.686 \pm 0.007 \\
1.634 \pm 0.011 \\
0.949 \pm 0.006\end{array}$ & $\begin{array}{l}0.079 \pm 0.008 \\
0.101 \pm 0.011 \\
0.124 \pm 0.015 \\
0.052 \pm 0.007 \\
0.075 \pm 0.011 \\
0.041 \pm 0.006\end{array}$ & $\begin{array}{l}0.490 \pm 0.024 \\
1.393 \pm 0.026 \\
2.583 \pm 0.029 \\
0.901 \pm 0.005 \\
2.091 \pm 0.009 \\
1.190 \pm 0.006\end{array}$ & $\begin{array}{l}0.120 \pm 0.018 \\
0.136 \pm 0.017 \\
0.161 \pm 0.019 \\
0.029 \pm 0.004 \\
0.051 \pm 0.006 \\
0.030 \pm 0.005\end{array}$ & $\begin{array}{l}0.39 \\
0.39 \\
0.39 \\
0.38 \\
0.39 \\
0.39\end{array}$ & $\begin{array}{l}1.52 \\
1.35 \\
1.30 \\
0.56 \\
0.68 \\
0.73\end{array}$ & $\begin{array}{l}0.001 \\
0.001 \\
0.001 \\
0.001 \\
0.001 \\
0.001\end{array}$ & $\begin{array}{l}0.017 \\
0.001 \\
0.001 \\
0.014 \\
0.001 \\
0.001\end{array}$ & $\begin{array}{l}0.001 \\
0.001 \\
0.001 \\
0.001 \\
0.001 \\
0.001\end{array}$ \\
\hline \multicolumn{10}{|c|}{ NGC1387 } \\
\hline $\begin{array}{l}U-B \\
U-V \\
U-I \\
B-V \\
B-I \\
V-I\end{array}$ & $\begin{array}{c}-0.019 \pm 0.017 \\
0.693 \pm 0.020 \\
1.624 \pm 0.023 \\
0.707 \pm 0.010 \\
1.642 \pm 0.012 \\
0.931 \pm 0.007\end{array}$ & $\begin{array}{l}0.084 \pm 0.011 \\
0.095 \pm 0.013 \\
0.114 \pm 0.014 \\
0.046 \pm 0.008 \\
0.058 \pm 0.006 \\
0.038 \pm 0.009\end{array}$ & $\begin{array}{l}0.446 \pm 0.030 \\
1.355 \pm 0.035 \\
2.505 \pm 0.044 \\
0.904 \pm 0.010 \\
2.056 \pm 0.017 \\
1.148 \pm 0.013\end{array}$ & $\begin{array}{l}0.128 \pm 0.017 \\
0.160 \pm 0.021 \\
0.196 \pm 0.022 \\
0.045 \pm 0.008 \\
0.079 \pm 0.009 \\
0.052 \pm 0.009\end{array}$ & $\begin{array}{l}0.49 \\
0.49 \\
0.49 \\
0.51 \\
0.49 \\
0.49\end{array}$ & $\begin{array}{l}1.52 \\
1.68 \\
1.72 \\
0.98 \\
1.36 \\
1.37\end{array}$ & $\begin{array}{l}0.001 \\
0.001 \\
0.001 \\
0.001 \\
0.001 \\
0.001\end{array}$ & $\begin{array}{l}0.041 \\
0.011 \\
0.005 \\
0.039 \\
0.002 \\
0.017\end{array}$ & $\begin{array}{l}0.001 \\
0.001 \\
0.001 \\
0.001 \\
0.001 \\
0.001\end{array}$ \\
\hline
\end{tabular}



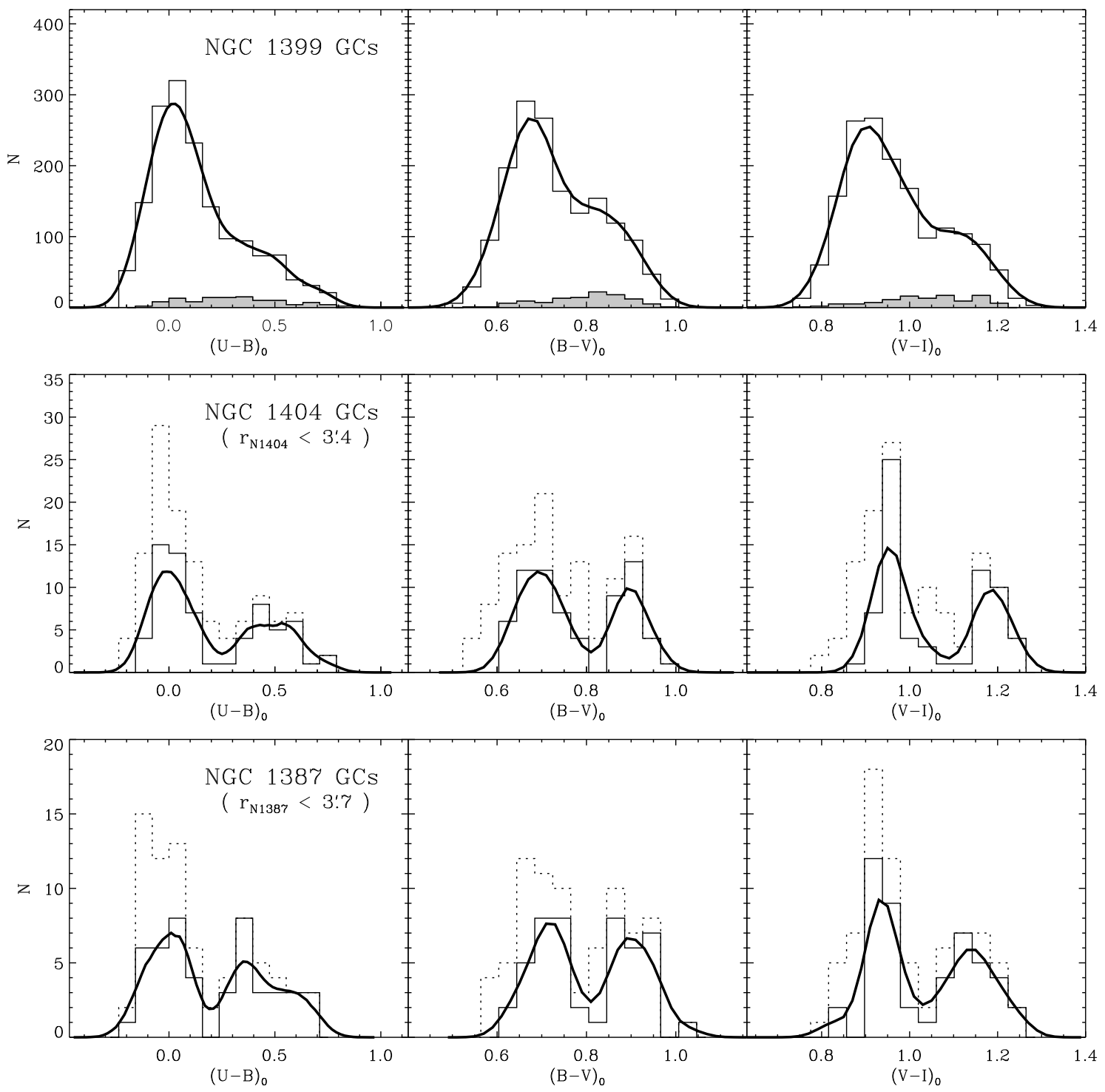

FIG. 12.- Color distributions of GCs in NGC 1399, NGC 1404, and NGC 1387. Thick solid lines are smoothed histograms with Gaussian kernels of $\sigma(U-B)=0.08, \sigma(B-V)=0.04$, and $\sigma(V-I)=0.04$, respectively. The $\sigma$ values are set to be comparable to the mean errors in each color. Filled gray histograms in the top panels show the color distributions of the brightest GCs $\left(V_{0}<21.5\right)$. Dotted histograms in the middle and bottom panels represent the color histograms of entire set of GCs within the extent of each galaxy, and solid histograms show the "corrected" distributions for the contamination by NGC 1399 GCs (see the text). 

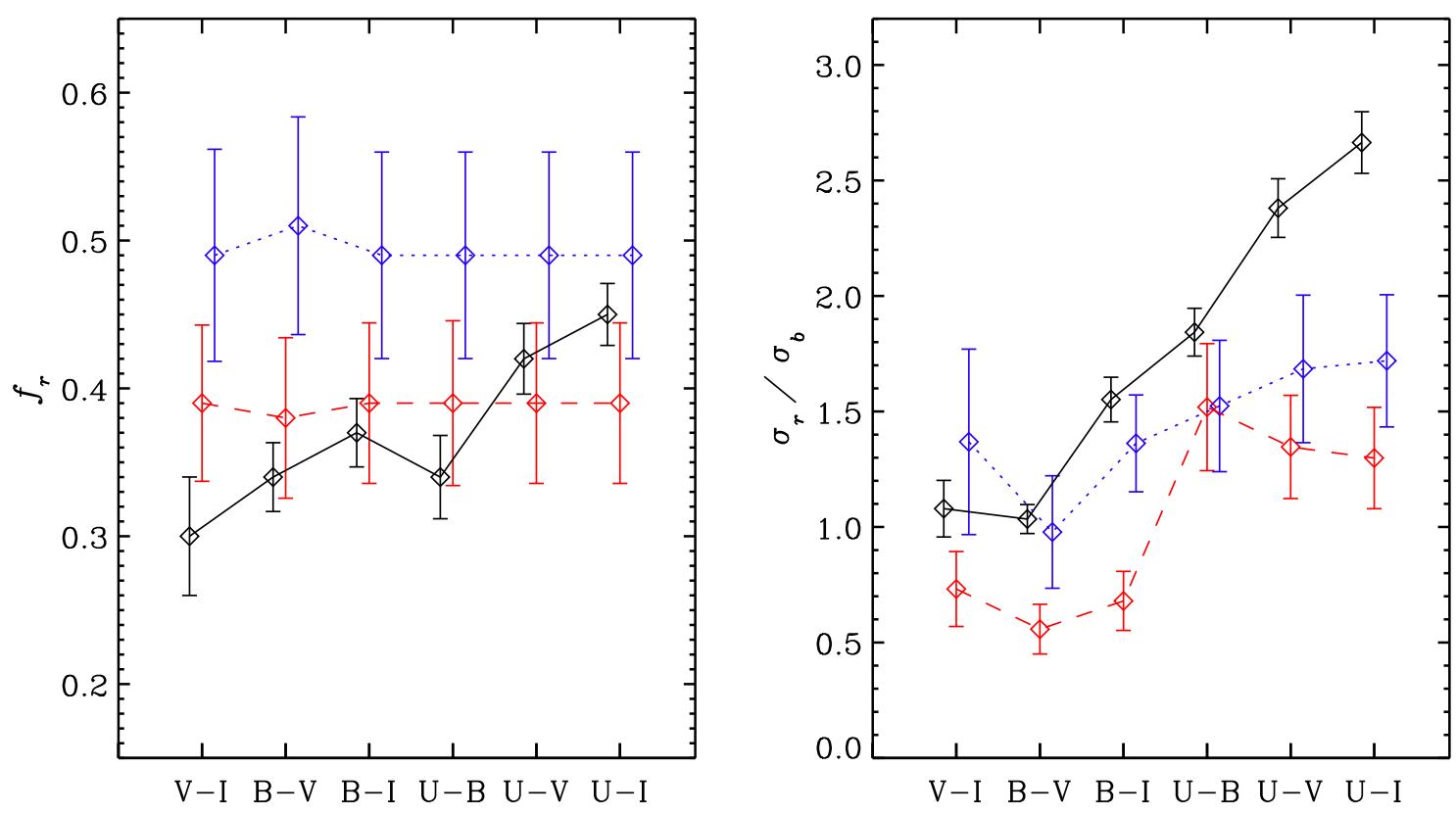

FIG. 13.- Variation in the red GC fractions (left) and the ratios of standard deviations between blue and red GC colors (right) for various colors. Black solid lines, red dashed lines, and blue dotted lines are for NGC 1399, NGC 1404, and NGC 1387, respectively. For NGC 1404 and NGC 1387, the values were derived from the corrected color distributions for the contribution of NGC 1399 GCs in Figure 12

are bluer at $(U-B)_{0} \simeq-0.8$ and foreground stars are at $(U-B)_{0}=-0.3$ and 1.1 close to both ends of the GC range. The $V_{0}-(B-V)_{0}$ CMD (right panel) shows that many of the background galaxies have similar colors to the GCs, making it difficult to discriminate GCs from galaxies in this color. The foreground star sequences, particularly on the red side, are more clearly separated from GCs than in $(U-B)_{0}$. This comparison corroborates that the use of various color combinations including $U$-band colors provides a powerful tool for discriminating GCs from other objects.

\subsection{Color-Magnitude Diagrams}

It is interesting to note that the CMDs do not show bimodality for GCs brighter than $V_{0} \sim 21.5$. The disappearance was previously reported for NGC 1399 (Ostrov et al. 1998; Dirsch et al. 2003; Blakeslee et al. 2012), a few galaxies in the Virgo Cluster (Mieske et al. 2006; Strader et al. 2006), and other giant galaxies (Harris et al. 2006; Faifer et al. 2011). The unimodal distribution is often explained by merging of blue and red sequences at the highest luminosities as a result of the blue tilt phenomenon (Harris et al. 2006), which is a trend for the blue GCs to have redder colors at higher luminosities. However, Ostrov et al. (1998) and Forte et al. (2007) have reported that there is no blue tilt in the GC system of NGC 1399. Interestingly, our data reveal that the number of blue GCs rapidly drops at the brightest magnitudes $\left(V_{0}<21.5\right)$. This suggests that the unimodal distribution of brightest GCs is not the consequence of the blue tilt, but instead owing to the relative scarcity of blue, brightest GCs.

\subsection{Color Distributions}

Figure 12 shows three representative color histograms of the entire GC system within the limiting radius in NGC 1399 (top), NGC 1404 (middle), and NGC 1387 (bottom). The GCs fainter than the limiting magnitudes and/or the ones located in the innermost region of each galaxy, where the completeness test is unreliable (see Section 2.3), are taken out from the subsequent analysis. The thick solid lines are smoothed histograms with Gaussian kernels. The filled histograms in the top row are for the brightest GCs, as mentioned in the previous section. For NGC 1399 (top row), GCs show bimodal distributions for all colors considered in agreement with previous findings in $C-T 1$ (Dirsch et al. 2003; Bassino et al. 2006a; Forte et al. 2007). For NGC 1404 (middle) and NGC 1387 (bottom), the dotted histograms are for all GCs within the limiting radii of the galaxies. The solid histograms represent "corrected" distributions, for which the contribution of NGC 1399 GCs is statistically subtracted from the original distributions and will be used in the further analysis. NGC 1404 GCs exhibit pronounced bimodality with an evident dip between two groups in all colors, consistent with the previous studies for NGC 1404's inner GCs by Grillmair et al. (1999) and Larsen et al. (2001). The clear separation between blue and red GCs for NGC 1387 GCs is also consistent with what was found in $C-T 1$ by Bassino et al. (2006b). Blue GCs are much less abundant in NGC 1404 and NGC 1387 than in NGC 1399.

A closer scrutiny of Figure 12 reveals that the exact morphologies of the GC color distributions change depending on colors considered. The most distinctive case is given by the $(U-B)_{0}$ distribution of NGC 1399 . Compared to the $(B-V)_{0}$ and $(V-I)_{0}$ histograms, the $(U-B)_{0}$ distribution exhibits more prominent blue GC peak and have red GCs with weaker peak and larger dispersion in color. The $(U-B)_{0}$ distribution shows spread in colors of red GCs that is nearly a factor of two larger than that of blue GCs, while the $(B-V)_{0}$ and $(V-I)_{0}$ histograms have similar variances for the red and blue GCs. The histograms for NGC 1404 and NGC 1387 also 
show changes in their shape depending on colors.

To be more quantitative in the bimodality analysis, we used the Gaussian Mixture Modeling (GMM) code by Muratov \& Gnedin (2010). Table 6 presents the results of the GMM analysis for six color combinations ( $U-$ $B)_{0},(U-V)_{0},(U-I)_{0},(B-V)_{0},(B-I)_{0}$, and $(V-$ $I)_{0}$ ) for the three galaxies. It gives the color index, the mean $(\mu)$, and the standard deviation $(\sigma)$ of blue and red GC colors, the red GC fraction $\left(f_{r}=N_{\text {red }} / N_{\text {total }}\right)$, and the ratio of the standard deviations between blue and red GCs $\left(\sigma_{r} / \sigma_{b}\right)$. The last three columns summarize the probabilities of preferring a unimodal distribution over a bimodal distribution ( $p$-values) derived based on the likelihood ratio test (LRT; $\chi^{2}$ ), on the separation of the means relative to their variances $(D D)$ and on the kurtosis of a distribution (kurt) 3

Figure 13 presents the results from the GMM analysis for all six color combinations. To quantify the shape of histograms, we use the red GC fractions (left) and the ratios of standard deviations between blue and red GC colors (right). NGC 1399 GC sample (black solid lines) does not include GCs around NGC 1404 and NGC 1387, and the GC sample of NGC 1404 (red dashed lines) and NGC 1387 (blue dotted) is corrected for NGC 1399 GC contribution. For NGC 1399, we find that both the numbers and dispersions of blue and red GCs change significantly depending on colors that are used. The trend is more evident when comparing the $U$-band colors with the rest. For NGC 1404 and NGC 1387, the interesting features emerge after the correction for the contribution of NGC 1399 GCs. First, the red GC fractions in all colors remain constant regardless of the colors, as expected in Figure 12 showing distinct separation between blue and red GCs. It is plausible that the early interactions of the galaxies with NGC 1399 have preferentially left the central, red GCs and later blue GCs have been accreted from outer region of NGC 1399. Second, the color dispersions vary significantly depending on colors, following a similar pattern to the case of NGC 1399 for $V-I, B-V$, and $B-I$. For $U$-band colors, however, the patterns are different in the sense that they tend to be constant within the errors. The variation of dispersion as a function of colors is not expected if the metallicity-to-color converting relations are straight. Instead, the slopes seem different between the blue and red parts of the metallicity-color relations and such an effect varies systematically from color to color (Yoon et al. 2006, 2011a,b, 2012; Cantiello \& Blakeslee 2007; Chies-Santos et al. 2012; Blakeslee et al. 2012).

\subsection{Radial Variations of Color Bimodality}

Figure 14 displays the color distributions as a function of galactocentric radius for GCs in NGC 1399. The subsequent analysis is only valid for NGC 1399 because small number statistics prohibit us making any firm statement on the radial variations of GC colors for the two fainter

\footnotetext{
3 Note that the kurtosis gives a necessary (but not sufficient) condition of bimodality, and it can be used only as an additional check of other statistics (Muratov \& Gnedin 2010). For all galaxies, $p\left(\chi^{2}\right)$ from the LRT is $\leq 0.01$ in all colors, indicating the bimodal distributions are preferred over the unimodal ones. The $p(D D)$ values lead to the same conclusion in all cases. The $p(k u r t)$ values support the bimodal distributions in most cases, but favor a unimodal distribution for $(U-B)_{0}$ of NGC 1399 with $p(k u r t)=0.860$.
}

galaxies after the correction for the NGC 1399 GC contamination. In order to investigate the radial variation of color bimodality, we set up a series of radial bins containing approximately equal number $(\sim 300)$ of GCs and perform the GMM analysis for each bin. We focus particularly on three main parameters of the GMM analysis that characterize the bimodal distributions; the number ratio between blue and red GCs, the mean colors of the groups, and their color dispersions.

Figure [15] shows the variations of color bimodality properties as a function of galactocentric radius. There is an obvious radial trend for the mean colors of both blue and red GCs getting bluer with increasing radius in all colors. The radial color gradient of red GCs is steeper than that of blue GCs. The red GC fraction in each color rapidly decreases with increasing galactocentric radius out to $r \simeq 4^{\prime}$, and it remains fairly constant beyond the point. There is a weak radial trend in GC color dispersions, in that the ratio between dispersions of blue and red GCs increases with radius.

\section{SUMMARY AND CONCLUSIONS}

We have performed wide-field $U B V I$ photometry of GCs in the central region of the Fornax cluster of galaxies with the Mosaic II CCD imager on the $4 \mathrm{~m}$ Blanco telescope at CTIO. This is one of the widest and deepest $U$-band studies on extragalactic GC systems. The reduction was carried out with the DAOPHOT II/ALLFRAME package. Using two-color diagrams and the magnitude cut of $M_{V} \leq-11$, a total of $2037 \mathrm{GC}$ candidates were selected among 12,134 objects detected in all four bands. Our estimate of the contamination by background galaxies and foreground stars in our sample is in the range of $9 \%-16 \%$. The sample is $U$-band limited, and the completeness of our photometry is $50 \%$ at $U_{0}=24.4$ according to our artificial star tests. We provide the $U B V I$ photometric catalog of the GC candidates online. For the GC systems of NGC 1399, NGC 1404, and NGC 1387, we have investigated the spatial distributions, CMDs, and color distributions. For NGC 1399 GCs, the radial variations in the properties of bimodality were examined as well. The main results are summarized as follows.

1. We find an asymmetric distribution of $\mathrm{GC}$ candidates around NGC 1399. The overabundance of GCs at the southeast side of the inner region and toward the directions of NGC 1404 and NGC 1387 in the outer region suggests that there were recent interactions of NGC 1399 with the galaxies and that NGC 1399 is not yet dynamically relaxed.

2. We specify the radial extent of each GC system at the radius where the surface density profile begins to depart from the de Vaucouleurs fit. The limiting radii are $\sim 3$ ! 4 for $\mathrm{NGC} 1404$ and $\sim 3$ !'7 for $\mathrm{NGC}$ 1387. The GC system of NGC 1399 is spatially extended beyond the field of view of our observation.

3. We show that the GC systems in the three galaxies exhibit bimodal color distributions in all the colors considered in this study. For the brightest GCs in NGC 1399, the bimodality becomes weak or disappear depending on colors. 


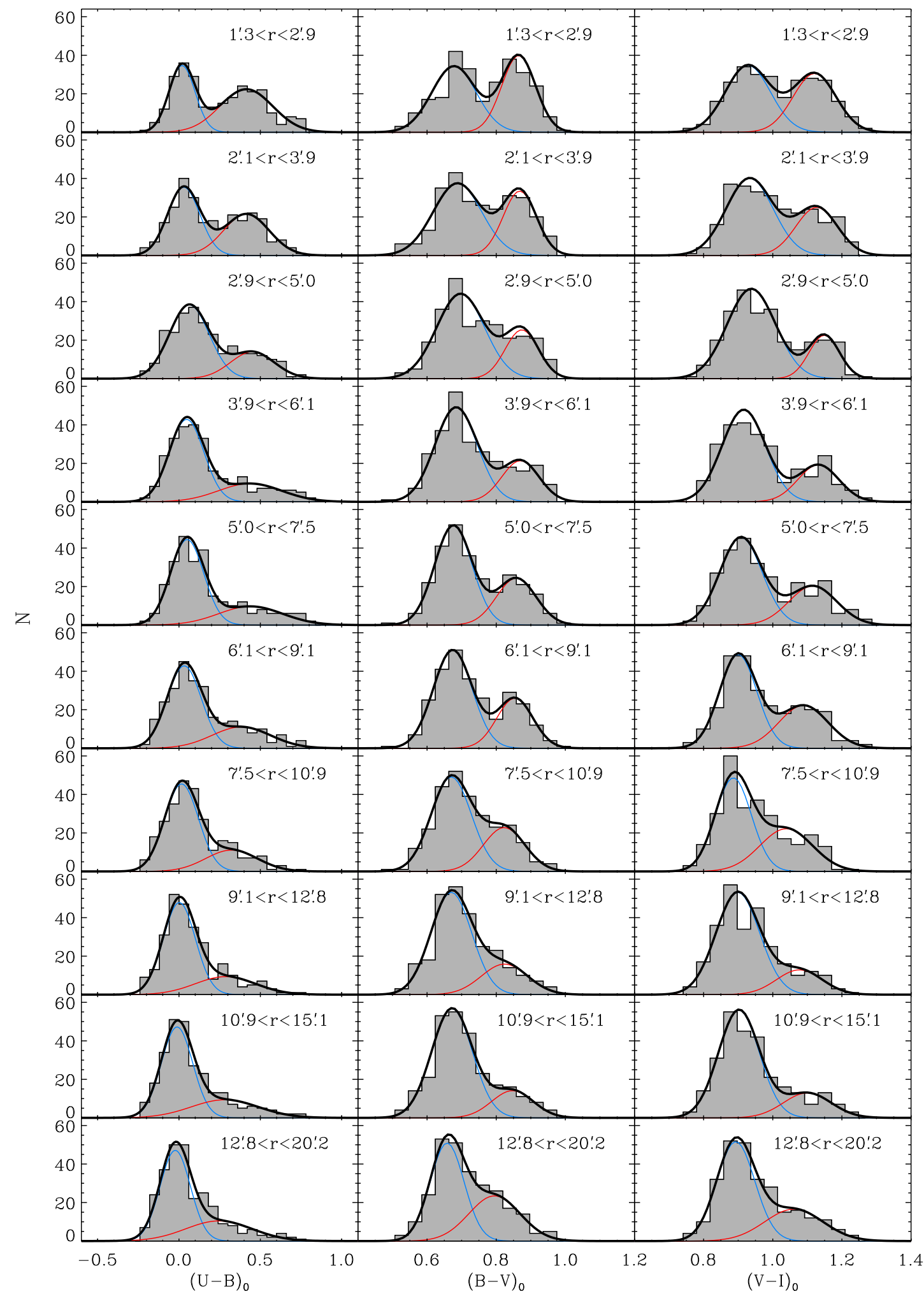

FiG. 14. - Histograms of $(U-B)_{0},(B-V)_{0}$, and $(V-I)_{0}$ colors (left, middle, and right columns) for NGC 1399 GCs in various radial bins indicated in the upper-right corner within each panel. The radial bins are defined to contain approximately equal numbers $(\sim 300)$ of GCs. The histograms are expressed by two (i.e., blue and red) Gaussian normal distributions based on GMM analysis. The blue, red, and black lines represent blue GCs, red GCs, and their sum, respectively. 

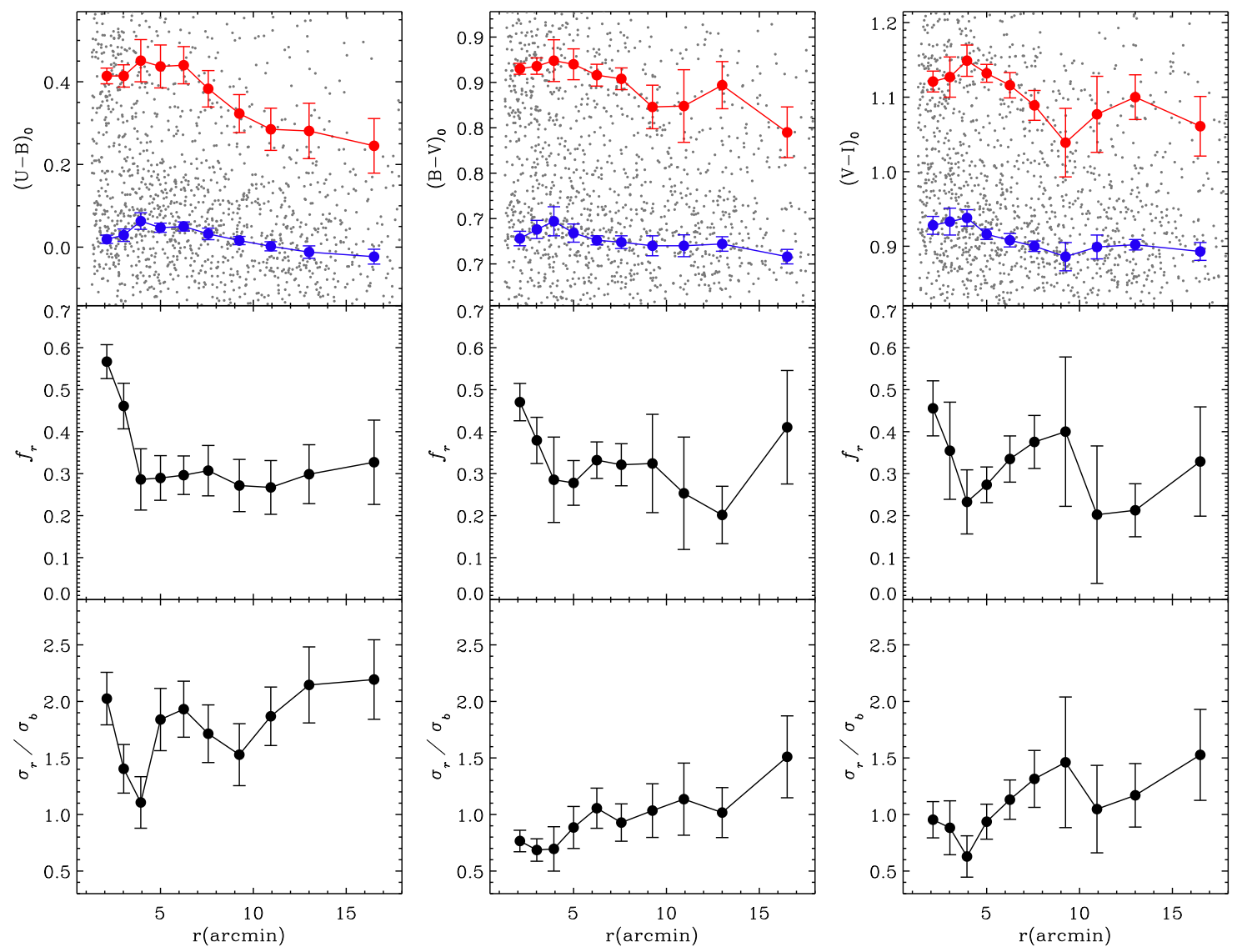

FIG. 15.- Parameters of bimodality in $(U-B)_{0},(B-V)_{0}$, and $(V-I)_{0}$ colors (left, middle, and right columns) as a function of galactocentric distance for NGC 1399 GCs. The top, middle, and bottom panels show the mean colors ( $\mu_{b}$ and $\mu_{r}$ for blue and red GCs), the red GC fraction $\left(f_{r}\right)$, and the ratios of color dispersions between blue and red GCs $\left(\sigma_{r} / \sigma_{b}\right)$, respectively. 
4. We find with GMM that the morphology of the color histograms changes significantly, depending on the colors used. The color dispersion of red GCs is larger than that of blue GCs in $U$-band colors by more than a factor of two, while the red and blue GCs show similar dispersions in $(B-V)_{0}$ and $(V-I)_{0}$. The number ratios between blue and red GCs vary depending on colors used as well. When corrected for the contribution of NGC 1399 GCs, the red GC fractions of NGC 1404 and NGC 1387 remain constant regardless of the colors used.

5. We suggest a possible scenario that could explain the presence of two discrete GC populations in NGC 1404 and NGC 1387: (1) the central, red GCs have been preferentially left through the early interactions of the galaxies with NGC 1399 and (2) later blue GCs have been accreted from outer region of NGC 1399.

6. We confirmed that the mean colors of blue and red GCs in NGC 1399 show a radial trend of becoming bluer with increasing galactocentric radius. The gradient of red GCs is steeper than that of blue GCs.

S.-J.Y. acknowledges support from International Exchange Program for University Researchers (NRF-2011013-C00031) and from Mid-career Research Program (No. 2012R1A2A2A01043870) through the National Research Foundation (NRF) of Korea grant funded by the Ministry of Education, Science and Technology (MEST), and support by the NRF of Korea to the Center for Galaxy Evolution Research (No. 2012-8-1743) and by the Korea Astronomy and Space Science Institute Research Fund 2011 and 2012. This work is partially supported by the KASI-Yonsei Joint Research Program (2011-2012) for the Frontiers of Astronomy and Space Science funded by the Korea Astronomy and Space Science Institute. This material is based upon work supported by AURA through the NSF under AURA Cooperative Agreement AST 0132798, as amended.

\section{REFERENCES}

Bassino, L. P., Faifer, F. R., Forte, J. C., et al. 2006, A\&A, 451, 789

Bassino, L. P., Richtler, T., \& Dirsch, B. 2006, MNRAS, 367, 156 Bekki, K., Forbes, D. A., Beasley, M. A., \& Couch, W. J. 2003, MNRAS, 344, 1334

Blakeslee, J. P., Cho, H., Peng, E. W., et al. 2012, ApJ, 746, 88

Blakeslee, J. P., Jordán, A., Mei, S., et al. 2009, ApJ, 694, 556

Bridges, T. J., Hanes, D. A., \& Harris, W. E. 1991, AJ, 101, 469

Cantiello, M., \& Blakeslee, J. P. 2007, ApJ, 669, 982

Cardelli, J. A., Clayton, G. C., \& Mathis, J. S. 1989, ApJ, 345, 245

Chies-Santos, A. L., Larsen, S. S., Cantiello, M., et al. 2012, A\&A, 539, A54

Chung, C., Yoon, S.-J., Lee, S.-Y., \& Lee, Y.-W. 2012, ApJS, 204, 3

Dirsch, B., Richtler, T., Geisler, D., et al. 2003, AJ, 125, 1908

Drinkwater, M. J., Gregg, M. D., \& Colless, M. 2001, ApJ, 548, 139

Faifer, F. R., Forte, J. C., Norris, M. A., et al. 2011, MNRAS, 416, 155

Fleming, D. E. B., Harris, W. E., Pritchet, C. J., \& Hanes, D. A. 1995, AJ, 109, 1044

Forbes, D. A., Beasley, M. A., Brodie, J. P., \& Kissler-Patig, M. 2001, ApJ, 563, 143

Forbes, D. A., Brodie, J. P., \& Grillmair, C. J. 1997, AJ, 113, 1652

Forbes, D. A., Grillmair, C. J., Williger, G. M., Elson, R. A. W., \& Brodie, J. P. 1998, MNRAS, 293, 325

Forte, J. C., Faifer, F., \& Geisler, D. 2005, MNRAS, 357, 56

Forte, J. C., Faifer, F., \& Geisler, D. 2007, MNRAS, 382, 1947

Grillmair, C. J., Forbes, D. A., Brodie, J. P., \& Elson, R. A. W. 1999, AJ, 117, 167

Grillmair, C. J., Freeman, K. C., Bicknell, G. V., et al. 1994, ApJ, 422, 9

Harris, W. E., \& van den Bergh, S. 1981, AJ, 86, 1627

Harris, W. E., Whitmore, B. C., Karakla, D., et al. 2006, ApJ, 636,90

Jordán, A., Blakeslee, J. P., Côté, P., et al. 2007, ApJS, 169, 213

Kissler-Patig, M., Brodie, J. P., Schroder, L. L., et al. 1998, AJ, 115,105

Kissler-Patig, M., Grillmair, C. J., Meylan, G., et al. 1999, AJ, 117,1206

Landolt, A. U. 1992, AJ, 104, 340
Larsen, S. S. 1999, A\&AS, 139, 393

Larsen, S. S., Brodie, J. P., Huchra, J. P., Forbes, D. A., \& Grillmair, C. J. 2001, AJ, 121, 2974

Liu, C., Peng, E. W., Jordán, A., et al. 2011, ApJ, 728, 116

Masters, K. L., Jordán, A., Côté, P., et al. 2010, ApJ, 715, 1419

Mieske, S., Jordán, A., Côté, P., et al. 2006, ApJ, 653, 193

Mieske, S., Jordán, A., Côté, P., et al. 2010, ApJ, 710, 1672

Monet, D. G., Levine, S. E., Canzian, B., et al. 2003, AJ, 125, 984

Muratov, A. L., \& Gnedin, O. Y. 2010, ApJ, 718, 1266

Ostrov, P. G., Forte, J. C., \& Geisler, D. 1998, AJ, 116, 2854

Ostrov, P., Geisler, D., \& Forte, J. C. 1993, AJ, 105, 1762

Paolillo, M., Fabbiano, G., Peres, G., \& Kim, D.-W. 2002, ApJ, 565,883

Richtler, T., Dirsch, B., Gebhardt, K., et al. 2004, AJ, 127, 2094

Richtler, T., Grebel, E. K., Domgoergen, H., Hilker, M., \& Kissler, M. 1992, A\&A, 264, 25

Robin, A. C., Reylé, C., Derrière, S., \& Picaud, S. 2003, A\&A, 409, 523

Schlegel, D. J., Finkbeiner, D. P., \& Davis, M. 1998, ApJ, 500, 525

Schuberth, Y., Richtler, T., Hilker, M., et al. 2010, A\&A, 513, A52

Secker, J. 1995, PASP, 107, 496

Stetson, P. B. 1990, PASP, 102, 932

Stetson, P. B. 1993, in IAU Colloq. 136: Stellar Photometry Current Techniques and Future Developments, ed. C. J. Butler \& I. Elliott (Cambridge: Cambridge Univ. Press), 291

Stetson, P. B. 1994, PASP, 106, 250

Stetson, P. B. 2000, PASP, 112, 925

Strader, J., Brodie, J. P., Spitler, L., \& Beasley, M. A. 2006, AJ, 132,2333

Valdes, F. G. 1998, in ASP Conf. Ser. 145, Astronomical Data Analysis Software and Systems VII, ed. R. Albrecht,

R. N. Hook, \& H. A. Bushhouse (San Francisco, CA: ASP), 53

Villegas, D., Jordán, A., Peng, E. W., et al. 2010, ApJ, 717, 603

Wagner, S., Richtler, T., \& Hopp, U. 1991, A\&A, 241, 399

Yoon, S.-J., Lee, S.-Y., Blakeslee, J. P., et al. 2011a, ApJ, 743, 150

Yoon, S.-J., Sohn, S., Kim, H.-S. et al. 2012, ApJ, submitted

Yoon, S.-J., Sohn, S. T., Lee, S.-Y., et al. 2011b, ApJ, 743, 149

Yoon, S.-J., Yi, S. K., \& Lee, Y.-W. 2006, Sci, 311, 1129 